\title{
SUBSTRATOS RENOVÁVEIS PARA PRODUÇÃO DE MUDAS DE Mimosa scabrella
}

\author{
Dagma Kratz ${ }^{1}$, Antonio Carlos Nogueira ${ }^{2}$, Ivar Wendling ${ }^{3}$, Paulo Vitor Dutra de Souza ${ }^{4}$ \\ ${ }^{1}$ Universidade Federal do Mato Grosso, Departamento de Engenharia Florestal, Cuiabá, Mato Grosso, Brasil - dagkratz@yahoo.com.br \\ ${ }^{2}$ Universidade Federal do Paraná, Departamento de Ciências Florestais, Curitiba, Paraná, Brasil - nogueira @ufpr.br \\ ${ }^{3}$ Empresa Brasileira de Pesquisa Agropecuária, Colombo, Paraná, Brasil - ivar@cnpf.embrapa.br \\ ${ }^{4}$ Universidade Federal do Rio Grande do Sul, Departamento de Horticultura e Silvicultura, Porto Alegre, Rio Grande do Sul, Brasil - \\ pvdsouza@ufrgs.br
}

Recebido para publicação: 24/03/2013 - Aceito para publicação: 19/11/2014

\begin{abstract}
Resumo
Objetivou-se neste estudo avaliar a viabilidade técnica de substratos renováveis à base de fibra de coco, casca de arroz carbonizada em diferentes granulometrias, biossólido e casca de pínus semidecomposta para a produção de mudas de Mimosa scabrella. Para tanto, foram formulados 41 tratamentos, contendo como testemunha substrato comercial à base de casca de pínus, os quais tiveram suas propriedades físicas e químicas avaliadas. Realizou-se semeadura direta em tubetes de $55 \mathrm{~cm}^{3}$, permanecendo por 120 dias em estufa de vidro e 30 dias na área de pleno sol. Observou-se que o substrato comercial à base de casca de pínus e substratos com diferentes combinações de casca de arroz carbonizada e fibra de coco apresentaram-se viáveis para produção de mudas de M. scabrella, enquanto que o componente biossólido mostrou-se inviável. O peneiramento da casca de arroz não se justifica, quando comparado com a casca de arroz carbonizada na sua forma íntegra. Quanto menor a agregação do substrato às raízes, maior a facilidade de retirada das mudas dos tubetes. A densidade, matéria orgânica, $\mathrm{pH}$, condutividade elétrica, salinidade e concentração de magnésio e fósforo influenciaram no crescimento das mudas.

Palavras-chave: Bracatinga; biossólido; casca de arroz carbonizada; fibra de coco; propriedades físicas.
\end{abstract}

\begin{abstract}
Renewable substrates in the seedling production of Mimosa scabrella. The objective of this study was to evaluate the technical feasibility of renewable-based substrates coconut fiber, rice hulls at different grain sizes, sludge and pine bark semi-decomposed for the production of seedlings of M. scabrella. To this end, 41 treatments were formulated, containing commercial substrate as a control the base of pine bark, where they were which were analyzed in relation to their physical and chemical properties. Held tillage in plastic tubes of $55 \mathrm{~cm}^{3}$ for 120 days in a greenhouse of glass and 30 days in the full sun. It was observed that the commercial substrate based on pine bark and the substrates based on different combinations of rice hulls and coconut fiber showed to be viable for production of seedlings of M. scabrella, while the component biosolid had to be unfeasible. Screening of rice husk is not justified when compared with the rice hulls in its entirety. The lower the aggregation in substrate, the easier removal of the seedlings of the tubes. The density, organic matter, $\mathrm{pH}$, electrical conductivity, salinity, magnesium and phosphorus influenced the growth of seedlings.
\end{abstract}

Keywords: Bracatinga; biosolid; rice hulls; coconut fiber; physical properties.

\section{INTRODUÇÃO}

Conhecida popularmente como bracatinga, Mimosa scabrella Benth é uma espécie pioneira, colonizadora de ambientes perturbados, onde a vegetação original foi derrubada ou teve influência antrópica que promoveu a abertura de clareiras (ROTTA; OLIVEIRA, 1981). Atualmente, a lenha continua sendo a principal utilização de sua madeira, isso porque a sua rotação é bastante curta, entre 6 e 8 anos, e não exige muitos tratos silviculturais. Segundo Weber (2007), a implantação e o manejo de bracatingais com o objetivo de produzir madeira para serraria ainda não é uma prática adotada em escala

FLORESTA, Curitiba, PR, v. 45, n. 2, p. 393 - 408, abr. / jun. 2015.

Kratz, D. et al.

ISSN eletrônico 1982-4688 / ISSN impresso 0015-3826

393

DOI: $10.5380 /$ rf.v45i2.31249 
comercial, no entanto, pesquisas indicam que essa espécie tem um grande potencial para produção de madeira com fins mais nobres ou de maior valor agregado.

As sementes de $M$. scabrella apresentam dormência tegumentar, que pode ser quebrada através do aquecimento pelo fogo, sendo esse um grande aliado na sua propagação e ocupação de novas áreas, através da quebra de dormência do banco de sementes presente no solo (EMPRESA BRASILEIRA DE PESQUISA AGROPECUÁRIA (EMBRAPA), 1988). Contudo, segundo Sturion (1981), a produção de mudas de M. scabrella justifica-se pelo fato do maior padrão de qualidade da muda formada no viveiro, pois as formadas no campo dependem de condições favoráveis de clima, solo, topografia, competição para germinação e desenvolvimento inicial.

Baseado na importância da qualidade da muda, muitos esforços têm sido realizados para melhorar a qualidade e reduzir os custos de produção das mesmas, e entre os fatores que influenciam na qualidade está o substrato (GONÇALVES; POGGIANI, 1996; CARNEIRO, 1995).

O avanço da tecnologia da produção de mudas proporcionou a substituição gradativa da terra de subsolo por outros materiais, principalmente renováveis, tendo como componentes cascas de árvores, grãos e compostos orgânicos, entre outros. A utilização desses materiais renováveis é de fundamental importância, haja vista o aumento da produção de mudas em todo o território nacional, que deve seguir os padrões de sustentabilidade. Dessa forma, devem-se aumentar os estudos em relação aos substratos, a fim de se apresentarem novas possibilidades de sua formulação, como a utilização de resíduos agroindustriais, florestais e urbanos.

Com base nisso, objetivou-se neste estudo avaliar a viabilidade técnica da utilização de substratos renováveis para a produção de mudas de $M$. scabrella e relacionar as características físicas e químicas deles com a qualidade das mudas produzidas.

\section{MATERIAL E MÉTODOS}

\section{Preparo e analise dos substratos}

Foi utilizado substrato comercial à base de casca de pínus semidecomposta (SC) e substratos baseados em misturas, ou de forma pura, dos seguintes componentes: biossólido - BIO (lodo de esgoto compostado), vermiculita média (VM), fibra de coco (FC) e três granulometrias de casca de arroz carbonizada (CAC), para a formulação de 41 substratos (Tabela 1).

O biosólido resultante do tratamento do esgoto pelo sistema anaeróbico foi obtido da Estação de Tratamento de Esgoto Atuba Sul, da Companhia de Saneamento do Paraná (SANEPAR), localizada em Curitiba, Paraná. Depois de exposto ao ar livre por 24 horas, passou por uma peneira de aço com malha de $10 \mathrm{~mm}$, para homogeneização das partículas.

Para obtenção das diferentes granulometrias de CAC, foram utilizadas três peneiras com malhas entre 1 e $2 \mathrm{~mm}$, de 0,5 a $1 \mathrm{~mm}$ e de 0,25 a $0,5 \mathrm{~mm}$.

Os materiais foram misturados com a adubação de base $\left(1,5 \mathrm{~kg} \cdot \mathrm{m}^{-3}\right.$ de substrato do fertilizante de liberação lenta de 6 meses (15:10:10, além de 3,5\% de Ca, 1,5\% de $\mathrm{Mg}, 3,0 \%$ de $\mathrm{S}, 0,05 \%$ de $\mathrm{Zn}, 0,02 \%$ de $\mathrm{B}, 0,05 \%$ de $\mathrm{Cu}, 0,1 \%$ de $\mathrm{Mn}, 0,5 \%$ de $\mathrm{Fe}$ e $0,004 \%$ de $\mathrm{Mo}$ ).

A caracterização física e química dos substratos foi realizada no Laboratório de Substratos do Departamento de Horticultura e Silvicultura da Universidade Federal do Rio Grande do Sul, conforme a metodologia descrita na Instrução Normativa $n^{\circ} 17$ do Ministério da Agricultura, Pecuária e Abastecimento (MAPA, 2007) e em Fermino (2003). A análise de nutrientes e matéria orgânica foi realizada no Laboratório de Solos da Embrapa Florestas, utilizando-se as metodologias descritas por Nogueira e Souza (2005).

\section{Produção de mudas}

O experimento foi instalado em março de 2010, no Laboratório de Propagação de Espécies Florestais da Embrapa Florestas, localizado em Colombo, Paraná, situado a 2519'17" de latitude S e 4909'39” de longitude W. O clima da região, de acordo com o Sistema Internacional de Köppen, é do tipo Cfb (clima subtropical úmido).

As sementes de $M$. scabrella passaram pelo processo de superação de dormência, em que se aplicou água à temperatura de $80^{\circ} \mathrm{C}$, deixando as sementes imersas por 18 horas (BIANCHETTI, 1981). Foram semeadas manualmente duas sementes por tubete de $55 \mathrm{~cm}^{3}(12 \times 3,5 \mathrm{~cm})$, acondicionados em bandejas metálicas, cobertas com uma fina camada $(0,5 \mathrm{~cm})$ de vermiculita média. Após a semeadura, as 
bandejas foram acondicionadas em estufa de vidro (três irrigações diárias de 10 min., com vazão de $144 \mathrm{~L} . h o r a^{-1}$ ), onde permaneceram por 120 dias, seguindo para área de rustificação (quatro irrigações diárias de 30 min., com vazão de 97 L.hora $^{-1}$ ), por 30 dias diretamente ao sol. Quatorze dias após a semeadura, foi realizado o raleamento, deixando como remanescente a muda mais centralizada no tubete e com maior crescimento da parte aérea.

Tabela 1. Material utilizado (\%) na formulação dos tratamentos (volume/volume).

Table 1. Material used (\%) in the formulation of treatments (volume/volume).

\begin{tabular}{|c|c|c|c|c|c|c|c|}
\hline Substrato & SC & BIO & VM & FC & $\begin{array}{c}\text { CAC } \\
\text { íntegra }\end{array}$ & $\begin{array}{c}\text { CAC } \\
0,5-1 \mathrm{~mm}\end{array}$ & $\begin{array}{c}\text { CAC } \\
0,25-0,5 \mathrm{~mm} \\
\end{array}$ \\
\hline 1 & 100 & & & & & & \\
\hline 2 & & & & & 100 & & \\
\hline 3 & & & & & & 100 & \\
\hline 4 & & & & & & & 100 \\
\hline 5 & & & 50 & & 50 & & \\
\hline 6 & & & & 10 & & 90 & \\
\hline 7 & & & & 25 & & 75 & \\
\hline 8 & & & & 10 & & & 90 \\
\hline 9 & & & & 25 & & & 75 \\
\hline 10 & & & & 80 & 20 & & \\
\hline 11 & & & & 70 & 30 & & \\
\hline 12 & & & & 60 & 40 & & \\
\hline 13 & & & & 50 & 50 & & \\
\hline 14 & & & & 40 & 60 & & \\
\hline 15 & & & & 30 & 70 & & \\
\hline 16 & & & & 20 & 80 & & \\
\hline 17 & & 50 & & & 50 & & \\
\hline 18 & & 40 & & & 60 & & \\
\hline 19 & & 30 & & & 70 & & \\
\hline 20 & & 20 & & & 80 & & \\
\hline 21 & & 10 & & & 90 & & \\
\hline 22 & & 50 & & 50 & & & \\
\hline 23 & & 50 & & 40 & 10 & & \\
\hline 24 & & 50 & & 30 & 20 & & \\
\hline 25 & & 50 & & 20 & 30 & & \\
\hline 26 & & 40 & & 50 & 10 & & \\
\hline 27 & & 40 & & 40 & 20 & & \\
\hline 28 & & 40 & & 30 & 30 & & \\
\hline 29 & & 40 & & 20 & 40 & & \\
\hline 30 & & 30 & & 50 & 20 & & \\
\hline 31 & & 30 & & 40 & 30 & & \\
\hline 32 & & 30 & & 30 & 40 & & \\
\hline 33 & & 30 & & 20 & 50 & & \\
\hline 34 & & 20 & & 50 & 30 & & \\
\hline 35 & & 20 & & 40 & 40 & & \\
\hline 36 & & 20 & & 30 & 50 & & \\
\hline 37 & & 20 & & 20 & 60 & & \\
\hline 38 & & 10 & & 50 & 40 & & \\
\hline 39 & & 10 & & 40 & 50 & & \\
\hline 40 & & 10 & & 30 & 60 & & \\
\hline 41 & & 10 & & 20 & 70 & & \\
\hline
\end{tabular}

SC: substrato florestal comercial à base de casca de pínus e vermiculita; BIO: biosólido; VM: vermiculita média; FC: fibra de coco; CAC: casca de arroz carbonizada original; CAC 0,5-1 mm: casca de arroz carbonizada com granulometria entre 0,5 e $1 \mathrm{~mm}$; CAC $0,25-0,5 \mathrm{~mm}$ : casca de arroz carbonizada com granulometria entre 0,25 e $0,5 \mathrm{~mm}$.

Aos 30 dias, aumentou-se o espaçamento entre as plantas, deixando o espaço de uma célula da bandeja, momento em que se iniciou a adubação de crescimento $(4 \mathrm{~g}$ de ureia, $3 \mathrm{~g}$ de superfosfato simples, 0,25 g de FTE BR 10 (7\% Zn, 4\% Fe, 4\% Mn, 0,1\% Mo, 2,5\% B, 0,8\% Cu) e $3 \mathrm{~g}$ de cloreto de potássio por litro de água), realizada a cada sete dias até os 120 dias, momento de transferência das mudas

FLORESTA, Curitiba, PR, v. 45, n. 2, p. 393 - 408, abr. / jun. 2015.

Kratz, D. et al.

ISSN eletrônico 1982-4688 / ISSN impresso 0015-3826 
para a área de pleno sol, onde se realizou adubação de rustificação (4 $\mathrm{g}$ de sulfato de amônio, $10 \mathrm{~g}$ de superfosfato simples, $4 \mathrm{~g}$ de cloreto de potássio e $1 \mathrm{~g}$ de FTE BR 10 por litro de água), realizada também a cada sete dias até os 150 dias. Para o preparo das adubações, os fertilizantes foram misturados com água e a solução resultante foi aplicada nas mudas com o uso de regador manual, aplicando-se aproximadamente $5 \mathrm{ml}$ por tubete.

O delineamento experimental utilizado foi o inteiramente casualizado, com cinco repetições de 20 plantas (unidade amostral) e 41 tratamentos.

Como características de avaliação da qualidade das mudas, foram mensuradas a altura da parte aérea e o diâmetro de colo a cada 30 dias até o final do experimento (150 dias). Para a medição, foi utilizada régua de precisão e paquímetro digital, respectivamente. Na última avaliação, foram feitas análises destrutivas em 10 plantas por repetição, selecionadas aleatoriamente, para avaliação da biomassa seca da parte aérea e radicial ( 48 horas em estufa a $65^{\circ} \mathrm{C}$ ), que foram pesadas em balança analítica de precisão $0,001 \mathrm{~g}$. Também se avaliou a facilidade de retirada do tubete e a agregação das raízes ao substrato. Para as avaliações de facilidade de retirada do tubete e agregação das raízes ao substrato, foi utilizada a metodologia descrita em Wendling et al. (2007). Esse método consiste em atribuir notas de zero a dez às variáveis, sendo zero a dificuldade máxima e dez a facilidade máxima de retirada das mudas após três batidas na parte superior (boca) do tubete. Quanto à agregação das raízes ao substrato, as mudas sem os tubetes foram soltas em queda livre a um metro do solo, e ao torrão foi atribuída uma nota de zero a dez, sendo zero para a muda totalmente esboroada e dez para o torrão íntegro (Figura 1).

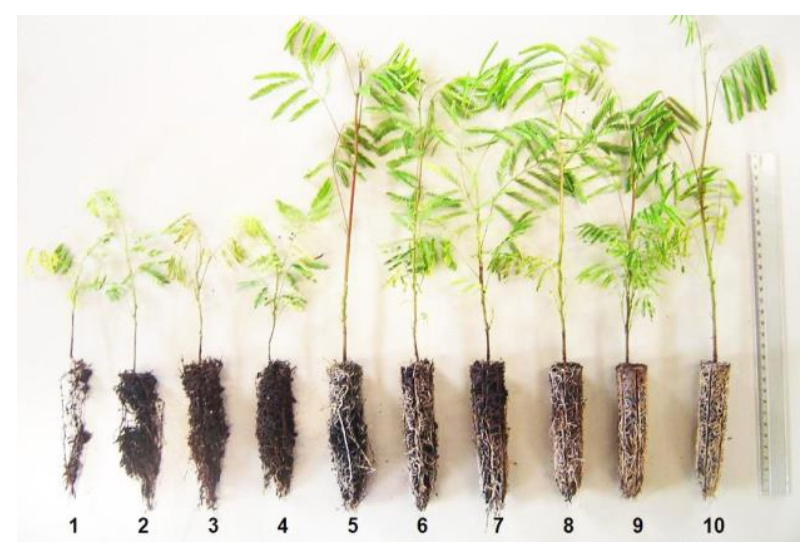

Figura 1. Índices de agregação das raízes ao substrato em mudas de $M$. scabrella.

Figure 1. Index of aggregation of the substrate roots of M. scabrella.

Também foram calculados os índices morfológicos: relação altura e diâmetro de colo (H/DC) e índice de qualidade de Dickson (IQD).

Os dados foram submetidos ao teste de Bartlett $(\mathrm{p}<0,05)$, a fim de verificar a condição de homogeneidade de variância, e, em seguida, a análise de variância (ANOVA) ( $<<0,01$ e p < 0,05), prosseguindo para o teste de Scott-Knott $(\mathrm{p}<0,01 \mathrm{e} \mathrm{p}<0,05)$, a fim de se observarem as diferenças entre as médias. Foi realizada a análise de correlação de Pearson entre as variáveis biométricas e as características físicas e químicas dos substratos, a fim de explicar quais propriedades tiveram maior influência nos resultados obtidos.

\section{RESULTADOS E DISCUSSÃO}

\section{Produção de mudas}

No crescimento em altura, as mudas de $M$. scabrella mostraram diferença significativa entre os substratos avaliados, para todos os períodos de avaliação (60, 90, 120 e 150 dias), sendo que os materiais renováveis CAC (casca de arroz carbonizada) e FC (fibra de coco), juntamente com SC (substrato comercial) e VM (vermiculita média), apresentaram-se adequados, enquanto que o componente BIO (biossólido) não apresentou viabilidade para o crescimento das mudas (Tabela 2). 
No que se refere ao componente $\mathrm{BIO}$, apenas o tratamento 10/20/70 (BIO/FC/CAC) apresentou altura de muda $(15,5 \mathrm{~cm})$ considerada apta ao plantio em campo, a qual, segundo Embrapa (1988) e Wendling e Dutra (2010), deve ser de no mínimo $15 \mathrm{~cm}$, e segundo Gonçalves et al. (2000) deve estar entre 20 e $35 \mathrm{~cm}$. Sendo assim, todos os tratamentos com BIO estão abaixo do padrão indicado (Tabela 2).

A resposta observada com $M$. scabrella ao BIO não é condizente ao verificado em outras pesquisas com diferentes espécies. Para Schinus terebinthifolius Raddi, a adição de BIO ao solo até a proporção de $35 \%$ ocasionou um incremento na altura das mudas (NÓBREGA et al., 2007). Em Eucalyptus grandis W. Hill ex Maiden, verificaram-se bons resultados com o uso do substrato CAC/BIO (50/50) (TRIGUEIRO; GUERRINI, 2003). E Kratz et al. (2013) verificaram aptidão técnica do uso de BIO como componente de substratos para a produção de Eucalyptus benthamii Maiden et Cambage.

Tabela 2. Altura aos 60, 90, 120 e 150 dias (H60, H90, H120 e H150), diâmetro aos 60, 90, 120 e 150 dias (D60, D90, D120 e D150), relação entre altura e diâmetro de colo aos 150 dias (H/D), biomassa seca aérea (BSA), biomassa seca radicial (BSR), índice de qualidade de Dickson (IQD), facilidade de retirada das mudas do tubete (FRT) e agregação das raízes ao substrato (AG) de mudas de M. scabrella produzidas em diferentes substratos.

Table 2. Height at 60, 90, 120 and 150 days (H60, H90, H120, H150), diameter 60, 90, 120 and 150 days (D 60, D90, D120 e D150), height to collar diameter ratio (H/D) at 150 days, shoot dry biomass (BSA), root dry biomass (BSR), Dickson quality index (IQD), ease of removal of seedlings of the tubes (FRT) and the substrate root aggregation (AG) of M. scabrella seedlings produced on different substrates.

\begin{tabular}{|c|c|c|c|c|c|c|c|c|c|c|c|c|c|c|}
\hline \multirow{2}{*}{ Substratos } & H60 & H90 & H120 & H150 & D 60 & D 90 & D120 & D150 & \multirow{2}{*}{ H/D } & BSA & BSR & \multirow{2}{*}{ IQD } & \multirow{2}{*}{ FRT } & \multirow{2}{*}{ AG } \\
\hline & \multicolumn{4}{|c|}{$(\mathbf{c m})$} & \multicolumn{4}{|c|}{$(\mathbf{m m})$} & & \multicolumn{2}{|c|}{ (g) } & & & \\
\hline $\mathrm{SC}$ & $6,6 \mathrm{a}$ & $7,8 \mathrm{a}$ & $16,6 \mathrm{a}$ & $21,2 \mathrm{a}$ & $1,0 \mathrm{a}$ & $1,4 \mathrm{a}$ & $1,9 \mathrm{a}$ & $2,5 \mathrm{a}$ & $8,6 \mathrm{a}$ & $0,8 \mathrm{a}$ & $0,4 \mathrm{~b}$ & $0,11 \mathrm{a}$ & $4 \mathrm{~d}$ & $8 \mathrm{a}$ \\
\hline CAC & $5,2 \mathrm{c}$ & $6,6 b$ & $10,0 \mathrm{c}$ & $12,6 \mathrm{~d}$ & $0,8 \mathrm{~b}$ & $1,2 \mathrm{~b}$ & $1,2 \mathrm{~d}$ & $1,6 \mathrm{~b}$ & $7,8 \mathrm{~b}$ & $0,4 \mathrm{c}$ & $0,3 \mathrm{c}$ & $0,07 \mathrm{~b}$ & $7 \mathrm{~b}$ & $5 \mathrm{c}$ \\
\hline $\mathrm{CAC}(0,5-1)$ & $5,1 \mathrm{c}$ & $6,2 \mathrm{c}$ & $10,1 \mathrm{c}$ & $13,7 \mathrm{~d}$ & $0,9 \mathrm{a}$ & $1,1 \mathrm{c}$ & $1,3 \mathrm{~d}$ & $1,6 \mathrm{~b}$ & $8,6 \mathrm{a}$ & $0,3 \mathrm{c}$ & $0,2 \mathrm{c}$ & $0,06 \mathrm{~b}$ & $7 \mathrm{~b}$ & $5 \mathrm{c}$ \\
\hline $\mathrm{CAC}(0,25-0,5)$ & $5,0 \mathrm{c}$ & $6,7 \mathrm{~b}$ & $13,2 \mathrm{~b}$ & $17,1 \mathrm{~b}$ & $0,9 \mathrm{a}$ & $1,1 \mathrm{c}$ & $1,4 \mathrm{c}$ & $1,8 \mathrm{~b}$ & 9,7 a & $0,4 \mathrm{c}$ & $0,2 \mathrm{~d}$ & $0,04 \mathrm{c}$ & $6 \mathrm{c}$ & $5 \mathrm{c}$ \\
\hline $50 \mathrm{CAC} / 50 \mathrm{VM}$ & $6,2 \mathrm{~b}$ & $7,3 \mathrm{~b}$ & $12,9 \mathrm{~b}$ & $17,2 \mathrm{~b}$ & $1,0 \mathrm{a}$ & $1,2 \mathrm{~b}$ & $1,7 \mathrm{~b}$ & $2,1 \mathrm{a}$ & $8,5 \mathrm{a}$ & $0,6 \mathrm{~b}$ & $0,4 \mathrm{a}$ & $0,11 \mathrm{a}$ & $5 \mathrm{c}$ & $7 \mathrm{~b}$ \\
\hline $10 \mathrm{FC} / 90 \mathrm{CAC}(0,5-1)$ & $5,2 \mathrm{c}$ & $7,0 \mathrm{~b}$ & $14,7 \mathrm{a}$ & $19,4 \mathrm{a}$ & $0,9 \mathrm{a}$ & $1,2 \mathrm{~b}$ & $1,7 \mathrm{~b}$ & $2,2 \mathrm{a}$ & $8,7 \mathrm{a}$ & $0,7 \mathrm{a}$ & $0,4 \mathrm{~b}$ & $0,10 \mathrm{a}$ & $4 \mathrm{~d}$ & $7 \mathrm{~b}$ \\
\hline $25 \mathrm{FC} / 75 \mathrm{CAC}(0,5-1)$ & $5,8 \mathrm{~b}$ & $7,1 \mathrm{~b}$ & $14,4 \mathrm{a}$ & $17,9 \mathrm{~b}$ & $0,9 \mathrm{a}$ & $1,3 \mathrm{a}$ & $1,7 \mathrm{~b}$ & $2,2 \mathrm{a}$ & $7,9 \mathrm{~b}$ & $0,7 \mathrm{a}$ & $0,4 \mathrm{a}$ & $0,12 \mathrm{a}$ & $3 \mathrm{~d}$ & $6 \mathrm{~b}$ \\
\hline $10 \mathrm{FC} / 90 \mathrm{CAC}(0,25-0,5)$ & $5,8 \mathrm{~b}$ & $7,1 \mathrm{~b}$ & $14,4 \mathrm{a}$ & $19,7 \mathrm{a}$ & $1,0 \mathrm{a}$ & $1,3 \mathrm{~b}$ & $1,7 \mathrm{~b}$ & $2,2 \mathrm{a}$ & 8,9 a & $0,7 \mathrm{a}$ & $0,5 \mathrm{a}$ & $0,12 \mathrm{a}$ & $5 \mathrm{c}$ & $7 \mathrm{~b}$ \\
\hline $25 \mathrm{FC} / 75 \mathrm{CAC}(0,25-0,5)$ & $6,1 \mathrm{~b}$ & 7,6 a & $16,3 \mathrm{a}$ & $21,4 \mathrm{a}$ & $1,0 \mathrm{a}$ & $1,3 \mathrm{a}$ & $2,0 \mathrm{a}$ & $2,4 \mathrm{a}$ & $8,8 \mathrm{a}$ & $0,8 \mathrm{a}$ & $0,5 \mathrm{a}$ & $0,13 \mathrm{a}$ & $3 \mathrm{~d}$ & $8 \mathrm{a}$ \\
\hline $80 \mathrm{FC} / 20 \mathrm{CAC}$ & $6,0 \mathrm{~b}$ & $7,2 \mathrm{~b}$ & $13,7 \mathrm{a}$ & $19,5 \mathrm{a}$ & $1,0 \mathrm{a}$ & $1,4 \mathrm{a}$ & $1,7 \mathrm{a}$ & $2,3 \mathrm{a}$ & $8,6 \mathrm{a}$ & $1,0 \mathrm{a}$ & $0,4 \mathrm{a}$ & $0,13 \mathrm{a}$ & $3 \mathrm{~d}$ & $8 \mathrm{a}$ \\
\hline $70 \mathrm{FC} / 30 \mathrm{CAC}$ & $6,5 \mathrm{a}$ & $7,8 \mathrm{a}$ & $15,5 \mathrm{a}$ & $20,2 \mathrm{a}$ & $1,0 \mathrm{a}$ & $1,4 \mathrm{a}$ & $1,9 \mathrm{a}$ & $2,3 \mathrm{a}$ & $9,1 \mathrm{a}$ & $0,8 \mathrm{a}$ & $0,4 \mathrm{a}$ & $0,11 \mathrm{a}$ & $4 \mathrm{~d}$ & $8 \mathrm{a}$ \\
\hline $60 \mathrm{FC} / 40 \mathrm{CAC}$ & $6,7 \mathrm{a}$ & $8,1 \mathrm{a}$ & $14,6 \mathrm{a}$ & $19,5 \mathrm{a}$ & $1,1 \mathrm{a}$ & $1,3 \mathrm{a}$ & $2,0 \mathrm{a}$ & $2,3 \mathrm{a}$ & $8,6 \mathrm{a}$ & $0,8 \mathrm{a}$ & $0,4 \mathrm{a}$ & $0,12 \mathrm{a}$ & $3 \mathrm{~d}$ & $8 \mathrm{a}$ \\
\hline $50 \mathrm{FC} / 50 \mathrm{CAC}$ & $7,0 \mathrm{a}$ & $8,2 \mathrm{a}$ & $15,2 \mathrm{a}$ & $19,4 \mathrm{a}$ & $1,0 \mathrm{a}$ & $1,5 \mathrm{a}$ & $2,0 \mathrm{a}$ & $2,3 \mathrm{a}$ & $8,4 \mathrm{a}$ & $0,8 \mathrm{a}$ & $0,4 \mathrm{a}$ & $0,12 \mathrm{a}$ & $2 \mathrm{~d}$ & $9 \mathrm{a}$ \\
\hline $40 \mathrm{FC} / 60 \mathrm{CAC}$ & $6,4 \mathrm{a}$ & $7,9 \mathrm{a}$ & $14,3 \mathrm{a}$ & $19,4 \mathrm{a}$ & $1,0 \mathrm{a}$ & $1,3 \mathrm{a}$ & $1,8 \mathrm{a}$ & $2,2 \mathrm{a}$ & $8,6 \mathrm{a}$ & $0,8 \mathrm{a}$ & $0,4 \mathrm{~b}$ & $0,11 \mathrm{a}$ & $5 \mathrm{c}$ & $8 a$ \\
\hline $30 \mathrm{FC} / 70 \mathrm{CAC}$ & $6,3 \mathrm{a}$ & 7,6 a & $14,3 \mathrm{a}$ & $18,4 \mathrm{a}$ & $1,0 \mathrm{a}$ & $1,3 \mathrm{a}$ & $1,8 \mathrm{a}$ & $2,4 \mathrm{a}$ & $7,7 \mathrm{~b}$ & $0,7 \mathrm{a}$ & $0,4 \mathrm{~b}$ & $0,11 \mathrm{a}$ & $3 \mathrm{~d}$ & $8 \mathrm{a}$ \\
\hline $20 \mathrm{FC} / 80 \mathrm{CAC}$ & $6,3 \mathrm{a}$ & $8,2 \mathrm{a}$ & $14,9 \mathrm{a}$ & $20,9 \mathrm{a}$ & $1,0 \mathrm{a}$ & $1,4 \mathrm{a}$ & $1,9 \mathrm{a}$ & $2,4 \mathrm{a}$ & $8,7 \mathrm{a}$ & $0,8 \mathrm{a}$ & $0,5 \mathrm{a}$ & $0,13 \mathrm{a}$ & $3 \mathrm{~d}$ & $8 \mathrm{a}$ \\
\hline $50 \mathrm{BIO} / 50 \mathrm{CAC}$ & $3,5 \mathrm{~d}$ & $4,0 \mathrm{~d}$ & $4,5 \mathrm{e}$ & $4,8 \mathrm{~g}$ & $0,6 \mathrm{c}$ & $0,7 \mathrm{e}$ & $0,9 \mathrm{e}$ & $0,9 \mathrm{e}$ & $5,4 \mathrm{c}$ & $0,1 \mathrm{e}$ & $0,1 \mathrm{e}$ & $0,03 \mathrm{c}$ & $10 \mathrm{a}$ & $1 \mathrm{f}$ \\
\hline $40 \mathrm{BIO} / 60 \mathrm{CAC}$ & $4,1 \mathrm{~d}$ & $5,5 \mathrm{c}$ & $7,3 \mathrm{e}$ & $9,1 \mathrm{e}$ & $0,6 \mathrm{c}$ & $0,9 \mathrm{~d}$ & $1,2 \mathrm{~d}$ & $1,2 \mathrm{~d}$ & $7,3 \mathrm{~b}$ & $0,2 \mathrm{~d}$ & $0,2 \mathrm{~d}$ & $0,04 \mathrm{c}$ & $9 \mathrm{a}$ & $2 \mathrm{f}$ \\
\hline $30 \mathrm{BIO} / 70 \mathrm{CAC}$ & $4,7 \mathrm{c}$ & $6,1 \mathrm{c}$ & $8,0 \mathrm{e}$ & $9,5 \mathrm{e}$ & $0,7 \mathrm{~b}$ & $1,1 \mathrm{c}$ & $1,3 \mathrm{c}$ & $1,4 \mathrm{c}$ & $6,8 \mathrm{c}$ & $0,2 \mathrm{~d}$ & $0,2 \mathrm{c}$ & $0,05 \mathrm{~b}$ & $8 \mathrm{a}$ & $3 \mathrm{~d}$ \\
\hline $20 \mathrm{BIO} / 80 \mathrm{CAC}$ & $5,3 \mathrm{c}$ & $6,7 \mathrm{~b}$ & $8,6 \mathrm{~d}$ & $9,8 \mathrm{e}$ & $0,8 \mathrm{~b}$ & $1,2 \mathrm{~b}$ & $1,5 \mathrm{c}$ & $1,5 \mathrm{c}$ & $6,8 \mathrm{c}$ & $0,3 \mathrm{c}$ & $0,2 \mathrm{c}$ & $0,06 \mathrm{~b}$ & $8 \mathrm{a}$ & $3 \mathrm{~d}$ \\
\hline $10 \mathrm{BIO} / 90 \mathrm{CAC}$ & $5,6 \mathrm{~b}$ & $7,3 \mathrm{~b}$ & $10,0 \mathrm{c}$ & $12,2 \mathrm{~d}$ & $0,8 \mathrm{~b}$ & $1,2 \mathrm{~b}$ & $1,5 \mathrm{c}$ & $1,6 \mathrm{~b}$ & $7,4 \mathrm{~b}$ & $0,4 \mathrm{c}$ & $0,3 \mathrm{c}$ & $0,07 \mathrm{~b}$ & $6 b$ & $4 \mathrm{~d}$ \\
\hline $50 \mathrm{BIO} / 50 \mathrm{FC}$ & $5,7 \mathrm{~b}$ & 7,6 a & $11,4 \mathrm{~b}$ & $14,7 \mathrm{c}$ & $0,9 \mathrm{a}$ & $1,3 \mathrm{a}$ & $1,6 \mathrm{~b}$ & $1,8 \mathrm{~b}$ & 8,1 a & $0,5 \mathrm{~b}$ & $0,3 \mathrm{~b}$ & $0,09 \mathrm{~b}$ & $6 \mathrm{~b}$ & $5 \mathrm{c}$ \\
\hline $50 \mathrm{BIO} / 40 \mathrm{FC} / 10 \mathrm{CAC}$ & $4,2 \mathrm{~d}$ & $5,1 \mathrm{~d}$ & $6,1 \mathrm{e}$ & $6,5 \mathrm{f}$ & $0,6 \mathrm{c}$ & $0,9 \mathrm{~d}$ & $1,1 \mathrm{~d}$ & $1,3 \mathrm{~d}$ & $5,2 \mathrm{c}$ & $0,3 \mathrm{c}$ & $0,2 \mathrm{~d}$ & $0,06 \mathrm{~b}$ & $10 \mathrm{a}$ & $1 \mathrm{f}$ \\
\hline $50 \mathrm{BIO} / 30 \mathrm{FC} / 20 \mathrm{CAC}$ & $5,0 \mathrm{c}$ & $5,1 \mathrm{~d}$ & $6,7 \mathrm{e}$ & $7,2 \mathrm{f}$ & $0,8 \mathrm{~b}$ & $0,9 \mathrm{~d}$ & $1,1 \mathrm{~d}$ & $1,2 \mathrm{~d}$ & $6,3 \mathrm{c}$ & $0,2 \mathrm{~d}$ & $0,1 \mathrm{e}$ & $0,03 \mathrm{c}$ & $10 \mathrm{a}$ & $2 \mathrm{f}$ \\
\hline $50 \mathrm{BIO} / 20 \mathrm{FC} / 30 \mathrm{CAC}$ & $4,1 \mathrm{~d}$ & $5,3 \mathrm{~d}$ & $7,0 \mathrm{e}$ & $7,0 \mathrm{f}$ & $0,7 \mathrm{~b}$ & $0,9 \mathrm{~d}$ & $1,2 \mathrm{~d}$ & $1,2 \mathrm{~d}$ & $5,8 \mathrm{c}$ & $0,2 \mathrm{~d}$ & $0,1 \mathrm{~d}$ & $0,05 \mathrm{~b}$ & $10 \mathrm{a}$ & $2 \mathrm{f}$ \\
\hline $40 \mathrm{BIO} / 50 \mathrm{FC} / 10 \mathrm{CAC}$ & $4,6 \mathrm{c}$ & $5,7 \mathrm{c}$ & $6,6 \mathrm{e}$ & $7,0 \mathrm{f}$ & $0,7 \mathrm{~b}$ & $0,9 \mathrm{~d}$ & $1,5 \mathrm{c}$ & $1,3 \mathrm{~d}$ & $5,4 \mathrm{c}$ & $0,2 \mathrm{~d}$ & $0,1 \mathrm{e}$ & $0,04 \mathrm{c}$ & $9 \mathrm{a}$ & $2 \mathrm{f}$ \\
\hline $40 \mathrm{BIO} / 40 \mathrm{FC} / 20 \mathrm{CAC}$ & $4,8 \mathrm{c}$ & $6,2 \mathrm{c}$ & $7,2 \mathrm{e}$ & $7,9 \mathrm{f}$ & $0,8 \mathrm{~b}$ & $1,0 \mathrm{c}$ & $1,2 \mathrm{~d}$ & $1,4 \mathrm{c}$ & $5,7 \mathrm{c}$ & $0,2 \mathrm{~d}$ & $0,2 \mathrm{~d}$ & $0,06 \mathrm{~b}$ & $9 \mathrm{a}$ & $3 \mathrm{f}$ \\
\hline $40 \mathrm{BIO} / 30 \mathrm{FC} / 30 \mathrm{CAC}$ & $5,4 \mathrm{c}$ & $5,7 \mathrm{c}$ & $7,5 \mathrm{e}$ & $7,9 \mathrm{f}$ & $0,8 \mathrm{~b}$ & $1,0 \mathrm{c}$ & $1,4 \mathrm{c}$ & $1,5 \mathrm{c}$ & $5,5 \mathrm{c}$ & $0,3 \mathrm{c}$ & $0,2 \mathrm{c}$ & $0,07 \mathrm{~b}$ & $9 \mathrm{a}$ & $4 \mathrm{~d}$ \\
\hline $40 \mathrm{BIO} / 20 \mathrm{FC} / 40 \mathrm{CAC}$ & $5,0 \mathrm{c}$ & $6,0 \mathrm{c}$ & $6,6 \mathrm{e}$ & $7,7 \mathrm{f}$ & $0,7 \mathrm{~b}$ & $1,1 \mathrm{c}$ & $1,2 \mathrm{~d}$ & $1,3 \mathrm{c}$ & $5,8 \mathrm{c}$ & $0,3 \mathrm{c}$ & $0,2 \mathrm{c}$ & $0,07 \mathrm{~b}$ & $8 \mathrm{a}$ & $3 \mathrm{~d}$ \\
\hline $30 \mathrm{BIO} / 50 \mathrm{FC} / 20 \mathrm{CAC}$ & $5,0 \mathrm{c}$ & $6,2 \mathrm{c}$ & $8,1 \mathrm{e}$ & $8,8 \mathrm{e}$ & $0,7 \mathrm{~b}$ & $1,0 \mathrm{c}$ & $1,4 \mathrm{c}$ & $1,5 \mathrm{~b}$ & $5,7 \mathrm{c}$ & $0,3 \mathrm{c}$ & $0,2 \mathrm{c}$ & $0,07 \mathrm{~b}$ & $9 \mathrm{a}$ & $3 \mathrm{~d}$ \\
\hline $30 \mathrm{BIO} / 40 \mathrm{FC} / 30 \mathrm{CAC}$ & $5,1 \mathrm{c}$ & $6,1 \mathrm{c}$ & $7,1 \mathrm{e}$ & $8,5 \mathrm{e}$ & $0,8 \mathrm{~b}$ & $1,0 \mathrm{c}$ & $1,4 \mathrm{c}$ & $1,4 \mathrm{c}$ & $5,9 \mathrm{c}$ & $0,3 \mathrm{c}$ & $0,2 \mathrm{c}$ & $0,07 \mathrm{~b}$ & $9 \mathrm{a}$ & $3 \mathrm{~d}$ \\
\hline $30 \mathrm{BIO} / 30 \mathrm{FC} / 40 \mathrm{CAC}$ & $5,1 \mathrm{c}$ & $6,2 \mathrm{c}$ & $8,7 \mathrm{~d}$ & $9,3 \mathrm{e}$ & $0,7 \mathrm{~b}$ & $1,1 \mathrm{c}$ & $1,4 \mathrm{c}$ & $1,4 \mathrm{c}$ & $6,6 \mathrm{c}$ & $0,2 \mathrm{~d}$ & $0,2 \mathrm{~d}$ & $0,05 \mathrm{~b}$ & $9 \mathrm{a}$ & $3 \mathrm{~d}$ \\
\hline $30 \mathrm{BIO} / 20 \mathrm{FC} / 50 \mathrm{CAC}$ & $5,5 \mathrm{~b}$ & $7,0 \mathrm{~b}$ & $9,2 \mathrm{~d}$ & $9,9 \mathrm{e}$ & $0,8 \mathrm{~b}$ & $1,4 \mathrm{a}$ & $1,5 \mathrm{c}$ & $1,7 \mathrm{~b}$ & $6,0 \mathrm{c}$ & $0,4 \mathrm{c}$ & $0,3 \mathrm{c}$ & $0,09 \mathrm{a}$ & $8 \mathrm{a}$ & $4 d$ \\
\hline $20 \mathrm{BIO} / 50 \mathrm{FC} / 30 \mathrm{CAC}$ & $5,9 \mathrm{~b}$ & $7,0 \mathrm{~b}$ & $7,6 \mathrm{e}$ & $8,5 \mathrm{e}$ & $0,9 \mathrm{a}$ & $1,4 \mathrm{a}$ & $1,5 \mathrm{c}$ & $1,5 \mathrm{c}$ & $5,9 \mathrm{c}$ & $0,2 \mathrm{~d}$ & $0,1 \mathrm{~d}$ & $0,05 \mathrm{~b}$ & $9 \mathrm{a}$ & $4 d$ \\
\hline $20 \mathrm{BIO} / 40 \mathrm{FC} / 40 \mathrm{CAC}$ & $5,6 \mathrm{~b}$ & $7,2 \mathrm{~b}$ & $8,5 \mathrm{~d}$ & $10,0 \mathrm{e}$ & $0,8 \mathrm{~b}$ & $1,3 \mathrm{a}$ & $1,5 \mathrm{c}$ & $1,7 \mathrm{~b}$ & $5,9 \mathrm{c}$ & $0,3 \mathrm{c}$ & $0,2 \mathrm{c}$ & 0,08 a & $7 \mathrm{~b}$ & $5 \mathrm{c}$ \\
\hline $20 \mathrm{BIO} / 30 \mathrm{FC} / 50 \mathrm{CAC}$ & $5,6 \mathrm{~b}$ & $6,5 \mathrm{~b}$ & $9,1 \mathrm{~d}$ & $10,1 \mathrm{e}$ & $0,8 \mathrm{~b}$ & $1,2 \mathrm{~b}$ & $1,6 \mathrm{~b}$ & $1,6 \mathrm{~b}$ & $6,4 \mathrm{c}$ & $0,3 \mathrm{c}$ & $0,2 \mathrm{c}$ & $0,07 \mathrm{~b}$ & $8 \mathrm{a}$ & $5 \mathrm{c}$ \\
\hline $20 \mathrm{BIO} / 20 \mathrm{FC} / 60 \mathrm{CAC}$ & $5,3 \mathrm{c}$ & $6,7 \mathrm{~b}$ & $8,7 \mathrm{~d}$ & $9,8 \mathrm{e}$ & $0,8 \mathrm{a}$ & $1,1 \mathrm{c}$ & $1,4 \mathrm{c}$ & $1,5 \mathrm{~b}$ & $6,4 \mathrm{c}$ & $0,3 \mathrm{c}$ & $0,2 \mathrm{c}$ & $0,07 \mathrm{~b}$ & $9 \mathrm{a}$ & $4 \mathrm{~d}$ \\
\hline $10 \mathrm{BIO} / 50 \mathrm{FC} / 40 \mathrm{CAC}$ & $6,0 \mathrm{~b}$ & $7,3 \mathrm{~b}$ & $10,1 \mathrm{c}$ & $11,2 \mathrm{~d}$ & $1,0 \mathrm{a}$ & $1,3 \mathrm{a}$ & $1,7 \mathrm{~b}$ & $1,7 \mathrm{~b}$ & $6,7 \mathrm{c}$ & $0,3 \mathrm{c}$ & $0,2 \mathrm{c}$ & $0,06 \mathrm{~b}$ & $8 \mathrm{a}$ & $5 \mathrm{c}$ \\
\hline $10 \mathrm{BIO} / 40 \mathrm{FC} / 50 \mathrm{CAC}$ & $5,8 \mathrm{~b}$ & $7,5 \mathrm{a}$ & $10,8 \mathrm{c}$ & $13,4 \mathrm{~d}$ & $0,9 \mathrm{a}$ & $1,2 \mathrm{~b}$ & $1,5 \mathrm{c}$ & $1,8 \mathrm{~b}$ & $7,6 \mathrm{~b}$ & $0,4 \mathrm{c}$ & $0,2 \mathrm{c}$ & $0,07 \mathrm{~b}$ & $7 \mathrm{~b}$ & $5 \mathrm{c}$ \\
\hline $10 \mathrm{BIO} / 30 \mathrm{FC} / 60 \mathrm{CAC}$ & $6,1 \mathrm{~b}$ & $7,2 \mathrm{~b}$ & $11,0 \mathrm{c}$ & $13,5 \mathrm{~d}$ & $1,0 \mathrm{a}$ & $1,2 \mathrm{~b}$ & $1,6 \mathrm{~b}$ & $1,8 \mathrm{~b}$ & $7,6 \mathrm{~b}$ & $0,5 \mathrm{~b}$ & $0,3 \mathrm{~b}$ & $0,09 \mathrm{a}$ & $7 \mathrm{~b}$ & $6 \mathrm{~b}$ \\
\hline $10 \mathrm{BIO} / 20 \mathrm{FC} / 70 \mathrm{CAC}$ & $6,5 \mathrm{a}$ & $8,3 \mathrm{a}$ & $12,9 \mathrm{~b}$ & $15,5 \mathrm{c}$ & $1,0 \mathrm{a}$ & $1,4 \mathrm{a}$ & $1,7 \mathrm{~b}$ & $2,1 \mathrm{a}$ & $7,6 \mathrm{~b}$ & $0,6 \mathrm{~b}$ & $0,3 \mathrm{~b}$ & $0,09 \mathrm{a}$ & $6 \mathrm{~b}$ & $7 \mathrm{~b}$ \\
\hline
\end{tabular}

Médias seguidas de uma mesma letra não diferem entre si pelo teste de Scott-Knott a 5\% de probabilidade de erro. SC: substrato comercial à base de casca de pínus e vermiculita; BIO: biossólido; VM: vermiculita média; FC: fibra de coco; CAC: casca de arroz carbonizada.

FLORESTA, Curitiba, PR, v. 45, n. 2, p. 393 - 408, abr. / jun. 2015.

Kratz, D. et al.

ISSN eletrônico 1982-4688 / ISSN impresso 0015-3826 
Na figura 2, pode-se visualizar claramente a diferença entre os tratamentos, com o grupo de substratos formulados à base de $\mathrm{CAC} / \mathrm{FC}$ e $\mathrm{SC}$ apresentando altura superior à dos substratos à base de $\mathrm{BIO}$ e a diminuição dos teores deste componente proporcionando maior crescimento das mudas.

A CAC peneirada apresentou-se viável tecnicamente quando combinada com a FC, porém não se diferenciou dos tratamentos formulados à base de $\mathrm{CAC}$ em sua forma íntegra, não justificando a utilização do peneiramento, devido à menor praticidade de preparo. A maior diferença entre os tratamentos começou a ocorrer a partir do terceiro mês, ficando mais acentuada com o passar do tempo. Nota-se também um baixo incremento em altura nas mudas que continham BIO em sua composição, apresentando uma estagnação no crescimento após o quarto mês, não justificando sua permanência no viveiro após esse período (Tabela 2).

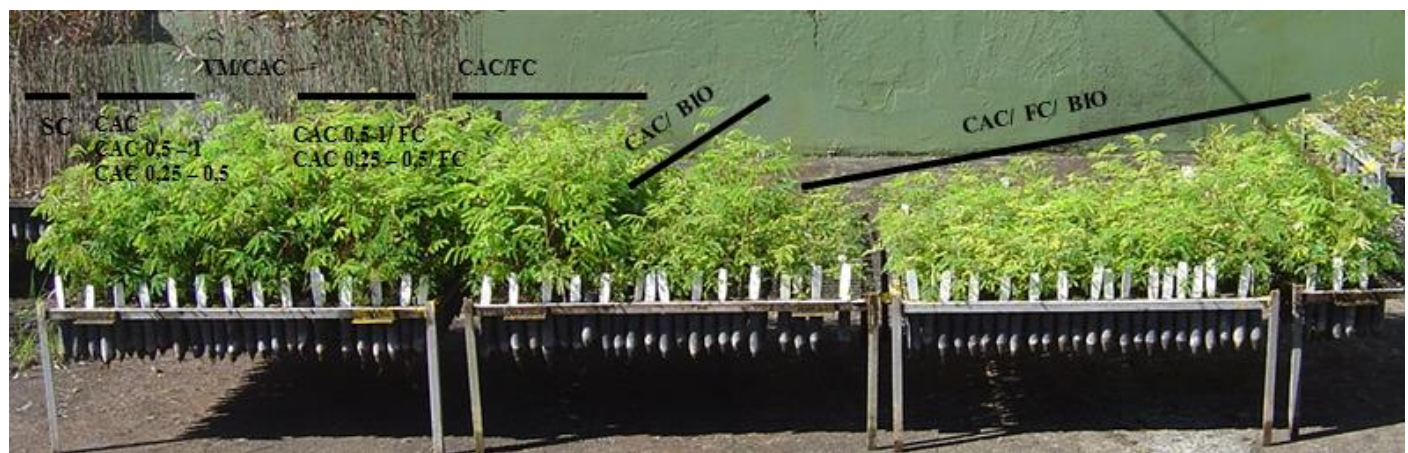

Figura 2. Foto ilustrativa das mudas de M. scabrella aos 150 dias, dispostas em ordem crescente de crescimento dentro de classes de tratamento.

Figure 2. Photo illustration of seedlings of $M$. scabrella to 150 days, arranged in ascending order of growth within classes of treatment.

É importante ressaltar que, aos 120 dias, alguns tratamentos já possibilitaram a obtenção de mudas aptas ao plantio definitivo, ou seja, com altura mínima de $15 \mathrm{~cm}$ (EMBRAPA, 1988; WENDLING; DUTRA, 2010), que são os seguintes: SC, com altura média de 16,6 cm; 25/75 de FC/CAC 0,25-0,5 mm, com 16,3 cm; e 70/30 e 50/50 de FC/CAC, com 15,5 e 15,2 cm, respectivamente (Tabela 2). Entretanto, levando em consideração a faixa de altura indicada por Gonçalves et al. (2000), faz-se necessária a permanência das mudas até os 150 dias, o que está fortemente relacionado às menores temperaturas do outono e início do inverno, época da condução do experimento, que podem prejudicar o crescimento das mudas.

Resultados semelhantes foram observados por Knapik et al. (2005) em mudas de M. scabrella, aos 120 dias, produzidas em substrato comercial à base de casca de pínus/fibra de coco $(70 / 30)(13 \mathrm{~cm})$.

Para o diâmetro de colo, observou-se comportamento semelhante ao da altura, apresentando diferença significativa entre os substratos avaliados para todos os períodos de avaliação. Os materiais renováveis CAC, assim como suas diferentes granulometrias, FC e o SC apresentaram-se adequados para produção de mudas dessa espécie, juntamente com o substrato $\mathrm{VM} / \mathrm{CAC}$, enquanto que o componente BIO não se mostrou apropriado (Tabela 2).

A diferenciação entre os tratamentos aumentou com a idade das mudas, ficando evidente que a presença de BIO provocou menor incremento em diâmetro nas mudas e que o aumento na proporção desse elemento proporcionou diminuição no crescimento em diâmetro de colo. Entre os substratos formulados à base de $\mathrm{BIO}$, apenas o tratamento contendo 10/20/70 de BIO/FC/CAC apresentou um bom crescimento (Tabela 2).

Resultados semelhantes foram observados por Knapik et al. (2005), com mudas de M. scabrella apresentando diâmetros variando de 1,1 a $2,1 \mathrm{~mm}$, e por Bonnet (2001), que mensurou 2,0 mm de diâmetro nas mudas produzidas à base de substrato comercial/biossólido compostado (70/30).

Nóbrega et al. (2007) observaram uma tendência de aumento no diâmetro de colo de mudas de Schinus terebinthifolius com a adição de BIO ao solo até a proporção de $37 \%$ e Trigueiro e Guerrini (2003) verificaram maior diâmetro de colo em mudas de Eucalyptus grandis nos substratos contendo 40 e $50 \%$ de BIO combinado com CAC. Dessa forma, BIO é viável para algumas espécies, porém não para a M. scabrella. 
Quanto à biomassa seca, o mesmo comportamento foi verificado para a parte aérea e radicial. $\mathrm{Na}$ tabela 2, observa-se que, para os componentes CAC, suas diferentes granulometrias e a FC apresentaramse adequadas para produção de mudas de $M$. scabrella, não se diferindo do SC e do substrato não renovável VM/CAC, enquanto que o componente BIO não foi considerado apto.

Trigueiro (2002) observou que, à medida que se diminuiu a dose de BIO no substrato, houve um efeito positivo no acúmulo de matéria seca de raiz até a proporção 50/50 (BIO/CAC) em mudas de Pinus taeda L. Já Cunha et al. (2006) obtiveram bons resultados com BIO inoculado com Rhizobium como substrato para a produção de mudas de Acacia mangium Wild, enquanto que esse componente combinado com subsolo na proporção 1:3 não apresentou um bom crescimento das mudas. Isso significa que o BIO empregado puro é a forma mais viável tecnicamente para produzir mudas de acácia, indicando que o substrato utilizado depende da espécie a ser produzida.

Quanto à variável facilidade de retirada das mudas do tubete, os que apresentaram maior facilidade foram os substratos que proporcionaram o menor crescimento das mudas (Tabela 2), apresentando correlação negativa com a altura $(R=-0,96)$, diâmetro de colo $(R=-0,95)$, biomassa seca aérea $(R=-0,96)$, biomassa seca radicial $(\mathrm{R}=-0,90)$ e agregação das raízes ao substrato $(\mathrm{R}=-0,94)$ (Tabela 3$)$.

A dificuldade apresentada na extração da muda do tubete está relacionada à maior massa de raízes, ocasionando maior compactação, o que dificulta a liberação do torrão. Kratz et al. (2013), trabalhando com os mesmos substratos, encontrou resultado contrário em mudas de Eucalyptus benthamii, em que as com maior enraizamento apresentaram também maior facilidade de retirada, visto que a massa das raízes da M. scabrella $(0,27 \mathrm{~g})$ foi superior à do Eucalyptus benthamii $(0,13 \mathrm{~g})$. Esse fato pode ser um indicativo de que o volume do tubete $\left(55 \mathrm{~cm}^{3}\right)$ utilizado para a produção das mudas de $M$. scabrella deveria ser maior, facilitando a retirada das mudas e resultando em menores danos ao sistema radicial. No entanto, o tubete pequeno poderá ser utilizado para a produção de mudas com menores alturas.

Trigueiro e Guerrini (2003) observaram resultados semelhantes, em que o SC apresentou maior dificuldade na extração do torrão, visto maior enraizamento de mudas de eucalipto nesse substrato quando comparado com os tratamentos que continham 80/20 e 60/40 de BIO/CAC. A facilidade de retirada das mudas do tubete é uma variável muito importante, visto que determina a rapidez de preparação das mudas no momento da expedição e diminui os danos no torrão formado (WENDLING et al., 2007).

Tabela 3. Correlações entre variáveis biométricas das mudas de M. scabrella. Altura (H150), diâmetro do colo (D150), biomassa seca aérea (BSA), biomassa seca radicial (BSR), facilidade de retirada das mudas dos tubetes (FRT) e agregação das raízes ao substrato (AG) aos 150 dias.

Table 3. Correlations between variables biometric seedlings M. scabrella. Height (H150), collar diameter (D150), shoot dry biomass (BSA), root dry biomass (BSR), ease of removal of the seedlings of the tubes (FRT) and the substrate root aggregation (AG) to 150 days.

\begin{tabular}{|c|c|c|c|c|c|c|}
\hline & H150 & D150 & BSA & BSR & AG & FRT \\
\hline H150 & $1,00^{* *}$ & & & & & \\
\hline D150 & $0,97^{* *}$ & $1,00^{* *}$ & & & & \\
\hline BSA & $0,97^{* *}$ & $0,98^{* *}$ & $1,00^{* *}$ & & & \\
\hline BSR & $0,90^{* *}$ & $0,90^{* *}$ & $0,93^{* *}$ & $1,00^{* * *}$ & & \\
\hline $\mathrm{AG}$ & $0,95^{* *}$ & $0,97^{\text {** }}$ & $0,96^{* *}$ & $0,91^{* *}$ & $1,00^{* *}$ & \\
\hline FRT & $-0,96^{* *}$ & $-0,95^{* *}$ & $-0,96^{* *}$ & $-0,90^{* *}$ & $-0,94^{* *}$ & $1,00^{* *}$ \\
\hline
\end{tabular}

Quanto à agregação das raízes ao substrato, os melhores resultados foram observados nos tratamentos com materiais renováveis à base de $\mathrm{CAC}, \mathrm{FC}$ e $\mathrm{SC}$, enquanto que os substratos contendo as maiores concentrações de $\mathrm{BIO}$ e as diferentes granulometrias de CAC, combinadas ou não com FC, e o substrato VM/CAC apresentaram maior esboroamento do torrão, devido à falta de agregação desses componentes, aliada à baixa massa radicial (Tabela 2).

Na tabela 3, verificam-se as correlações existentes entre a agregação das raízes ao substrato e à altura $(R=0,96)$, diâmetro $(R=0,95)$, biomassa seca aérea $(R=0,96)$, biomassa seca radicial $(R=0,90)$, que podem ser visivelmente observadas na figura 1. Resultados semelhantes foram encontrados por Trigueiro (2002) com mudas de Pinus, em que aquelas com enraizamento mais vigoroso apresentaram torrões mais firmes. Essa avaliação, segundo Wendling et al. (2007), é de grande importância, pois

FLORESTA, Curitiba, PR, v. 45, n. 2, p. 393 - 408, abr. / jun. 2015.

Kratz, D. et al.

ISSN eletrônico 1982-4688 / ISSN impresso 0015-3826 
informa a capacidade que o substrato tem de não se esboroar no momento da retirada do tubete e no plantio definitivo, além do fato de que, quanto maior a agregação, mais fácil se tornam todas as etapas posteriores de transporte e plantio.

Quanto à relação H/DC, os maiores valores foram observados nas mudas produzidas nos substratos renováveis formulados a partir de FC combinada com CAC em sua forma íntegra e nas diferentes granulometrias, não diferindo do SC, assim como no tratamento VM/CAC (Tabela 2). Conforme a indicação de Carneiro (1995), a relação H/DC deve situar-se entre 5,4 e 8,1. Dessa forma, apenas os tratamentos inferiores em relação à altura, diâmetro e biomassa enquadram-se dentro da faixa considerada adequada (Tabela 2). Logo, deve-se ficar atento à faixa recomendada, visto que ela pode não ser a mais adequada para a espécie em questão, pois as mudas produzidas nos substratos com maior relação H/DC são também as que apresentaram maior aptidão ao plantio em campo, visualmente e com base na agregação do sistema radicular ao substrato.

Bonnet (2001) observou uma relação H/DC de 10,7 em mudas de M. scabrella produzidas em substratos contendo $30 \%$ de lodo compostado e $70 \%$ de substrato comercial, enquanto que o substrato com $30 \%$ de lodo alcalinizado apresentou H/DC de 4,6, corroborando os resultados encontrados neste estudo.

Para o IQD, como para as outras características avaliadas, os melhores tratamentos foram aqueles formulados à base dos componentes renováveis CAC e suas diferentes granulometrias, combinadas com FC, e SC, assim como o tratamento não renovável VM/CAC (Tabela 2). Vários autores citam um valor mínimo de 0,20 para o IQD, de modo que nenhum dos tratamentos estudados atingiu o valor mínimo recomendado (Tabela 2). Porém deve-se lembrar que esse índice, segundo Gomes e Paiva (2004), foi desenvolvido para mudas de Picea glauca (L.) Karst. e Pseudotsuga menziessi (Mirbel) Franco, podendo não ser aplicado para mudas de M. scabrella.

\section{Análise de substratos}

Observa-se na tabela 4 que nenhum dos substratos analisados ultrapassou o limite máximo de densidade $\left(>500 \mathrm{~kg} \cdot \mathrm{m}^{-3}\right)$, porém a maioria apresentou densidade abaixo da recomendada $\left(<250 \mathrm{~kg} \cdot \mathrm{m}^{-3}\right)$, segundo Gonçalves e Poggiani (1996).

Nos substratos com densidade considerada baixa, as mudas apresentaram um bom crescimento, não sendo esse um fator limitante, como pode ser observado com as correlações significativas negativas encontradas: altura $(R=-0,65)$, diâmetro de colo $(R=-0,67)$, biomassa seca aérea $(R=-0,64)$, biomassa seca radicial $(R=-0,64)$, agregação das raízes ao substrato $(R=-0,75)$; e correlação positiva com a facilidade de retirada das mudas dos tubetes $(\mathrm{R}=0,65)$ (Tabela 5). A densidade do substrato é uma característica de grande importância na qualidade das mudas, podendo interferir no desenvolvimento das raízes, já que valores elevados podem promover maior impedância mecânica.

A porosidade total apresentou baixa variação entre os diferentes tratamentos, diferentemente da macro e microporosidade. Os substratos à base de FC e BIO apresentaram maior microporosidade e teor de água facilmente disponível, enquanto que o acréscimo de CAC ocasionou um aumento na macroporosidade e diminuição no teor de água facilmente disponível (Tabela 4). Esse resultado corrobora Gonçalves e Poggiani (1996), os quais afirmam que materiais com baixa densidade, como materiais incinerados, elevam a macroporosidade das misturas e reduzem a capacidade de retenção de água do substrato. Corrobora também o estudo realizado por Schmitz et al. (2002), os quais observaram que a adição de CAC à turfa reduziu a retenção de água, amenizando os problemas com excesso de umidade. No entanto, altas proporções de CAC tornam-se inviáveis, em virtude do alto consumo de água para irrigação (GUERRINI; TRIGUEIRO, 2004).

Noguera et al. (2000), analisando as propriedades físicas e químicas de turfa e FC, comprovaram que esses elementos apresentam características parecidas. Dessa forma, a FC tem potencial para substituir a turfa, visto que esta não é renovável, apresentando porosidade total média de $95,9 \%$, macroporosidade de 45,3\%, água facilmente disponível de $18,6 \%$ e pH 5,73.

No que se refere à porosidade total, a maioria dos substratos está dentro da faixa considerada adequada (75 a 85\%) por Gonçalves e Poggiani (1996) (Tabela 4). Essa característica, segundo Kämpf (2005), é de fundamental importância para o crescimento das plantas, visto que a alta concentração de raízes formadas nos recipientes exige elevado fornecimento de oxigênio e rápida remoção do gás carbônico formado. 
Tabela 4. Resultados da análise física e dos substratos: densidade aparente (Da), porosidade total (Pt), macroporosidade (Macro), microporosidade (Micro) e água facilmente disponível (AFD).

Table 4. Results of bulk density (Da), total porosity (PT), macroporosity (Macro) and microporosity (Micro) and readily available water (AFD) of the substrates evaluated.

\begin{tabular}{|c|c|c|c|c|c|}
\hline \multirow{2}{*}{ Substratos } & \multirow{2}{*}{$\underset{\mathrm{kg} \cdot \mathrm{m}^{3}}{\mathrm{Da}}$} & $\mathbf{P t}$ & Macro & Micro & AFD \\
\hline & & \multicolumn{4}{|c|}{ Natro } \\
\hline $\mathrm{SC}$ & 398,00 & 79,00 & 19,00 & 60,00 & 24,00 \\
\hline $\mathrm{CAC}$ & 101,00 & 72,00 & 57,00 & 15,00 & 6,00 \\
\hline $\mathrm{CAC}(0,5-1)$ & 200,00 & 85,00 & 21,00 & 64,00 & 45,00 \\
\hline $\mathrm{CAC}(0,25-0,5)$ & 239,00 & 80,00 & 11,00 & 69,00 & 45,00 \\
\hline $50 \mathrm{CAC} / 50 \mathrm{VM}$ & 183,00 & 72,00 & 37,00 & 35,00 & 9,00 \\
\hline $10 \mathrm{FC} / 90 \mathrm{CAC}(0,5-1)$ & 180,00 & 85,00 & 23,00 & 62,00 & 42,00 \\
\hline $25 \mathrm{FC} / 75 \mathrm{CAC}(0,5-1)$ & 174,00 & 85,00 & 24,00 & 61,00 & 41,00 \\
\hline $10 \mathrm{FC} / 90 \mathrm{CAC}(0,25-0,5)$ & 237,00 & 83,00 & 15,00 & 68,00 & 42,00 \\
\hline $25 \mathrm{FC} / 75 \mathrm{CAC}(0,25-0,5)$ & 204,00 & 83,00 & 11,00 & 72,00 & 45,00 \\
\hline $80 \mathrm{FC} / 20 \mathrm{CAC}$ & 89,00 & 85,00 & 36,00 & 49,00 & 20,00 \\
\hline $70 \mathrm{FC} / 30 \mathrm{CAC}$ & 86,00 & 84,00 & 38,00 & 46,00 & 18,00 \\
\hline $60 \mathrm{FC} / 40 \mathrm{CAC}$ & 89,00 & 86,00 & 45,00 & 41,00 & 17,00 \\
\hline $50 \mathrm{FC} / 50 \mathrm{CAC}$ & 96,00 & 90,00 & 57,00 & 33,00 & 13,00 \\
\hline $40 \mathrm{FC} / 60 \mathrm{CAC}$ & 93,00 & 90,00 & 55,00 & 35,00 & 14,00 \\
\hline $30 \mathrm{FC} / 70 \mathrm{CAC}$ & 96,00 & 90,00 & 54,00 & 36,00 & 15,00 \\
\hline $20 \mathrm{FC} / 80 \mathrm{CAC}$ & 101,00 & 89,00 & 62,00 & 27,00 & 12,00 \\
\hline $50 \mathrm{BIO} / 50 \mathrm{CAC}$ & 389,00 & 82,00 & 36,00 & 46,00 & 15,00 \\
\hline $40 \mathrm{BIO} / 60 \mathrm{CAC}$ & 362,00 & 84,00 & 40,00 & 44,00 & 16,00 \\
\hline $30 \mathrm{BIO} / 70 \mathrm{CAC}$ & 289,00 & 82,00 & 50,00 & 32,00 & 10,00 \\
\hline $20 \mathrm{BIO} / 80 \mathrm{CAC}$ & 229,00 & 86,00 & 56,00 & 30,00 & 12,00 \\
\hline $10 \mathrm{BIO} / 90 \mathrm{CAC}$ & 184,00 & 90,00 & 65,00 & 25,00 & 10,00 \\
\hline $50 \mathrm{BIO} / 50 \mathrm{FC}$ & 338,00 & 79,00 & 15,00 & 64,00 & 23,00 \\
\hline $50 \mathrm{BIO} / 40 \mathrm{FC} / 10 \mathrm{CAC}$ & 359,00 & 79,00 & 20,00 & 59,00 & 21,00 \\
\hline $50 \mathrm{BIO} / 30 \mathrm{FC} / 20 \mathrm{CAC}$ & 375,00 & 81,00 & 23,00 & 58,00 & 20,00 \\
\hline $50 \mathrm{BIO} / 20 \mathrm{FC} / 30 \mathrm{CAC}$ & 381,00 & 78,00 & 24,00 & 54,00 & 19,00 \\
\hline $40 \mathrm{BIO} / 50 \mathrm{FC} / 10 \mathrm{CAC}$ & 298,00 & 84,00 & 21,00 & 63,00 & 23,00 \\
\hline $40 \mathrm{BIO} / 40 \mathrm{FC} / 20 \mathrm{CAC}$ & 319,00 & 85,00 & 26,00 & 59,00 & 21,00 \\
\hline $40 \mathrm{BIO} / 30 \mathrm{FC} / 30 \mathrm{CAC}$ & 319,00 & 84,00 & 30,00 & 54,00 & 19,00 \\
\hline $40 \mathrm{BIO} / 20 \mathrm{FC} / 40 \mathrm{CAC}$ & 330,00 & 84,00 & 33,00 & 51,00 & 18,00 \\
\hline $30 \mathrm{BIO} / 50 \mathrm{FC} / 20 \mathrm{CAC}$ & 238,00 & 82,00 & 26,00 & 56,00 & 21,00 \\
\hline $30 \mathrm{BIO} / 40 \mathrm{FC} / 30 \mathrm{CAC}$ & 267,00 & 84,00 & 29,00 & 55,00 & 19,00 \\
\hline $30 \mathrm{BIO} / 30 \mathrm{FC} / 40 \mathrm{CAC}$ & 270,00 & 86,00 & 39,00 & 47,00 & 17,00 \\
\hline $30 \mathrm{BIO} / 20 \mathrm{FC} / 50 \mathrm{CAC}$ & 276,00 & 86,00 & 41,00 & 45,00 & 16,00 \\
\hline $20 \mathrm{BIO} / 50 \mathrm{FC} / 30 \mathrm{CAC}$ & 218,00 & 87,00 & 36,00 & 51,00 & 19,00 \\
\hline $20 \mathrm{BIO} / 40 \mathrm{FC} / 40 \mathrm{CAC}$ & 229,00 & 87,00 & 38,00 & 49,00 & 18,00 \\
\hline $20 \mathrm{BIO} / 30 \mathrm{FC} / 50 \mathrm{CAC}$ & 213,00 & 89,00 & 45,00 & 44,00 & 17,00 \\
\hline $20 \mathrm{BIO} / 20 \mathrm{FC} / 60 \mathrm{CAC}$ & 234,00 & 89,00 & 49,00 & 40,00 & 14,00 \\
\hline $10 \mathrm{BIO} / 50 \mathrm{FC} / 40 \mathrm{CAC}$ & 163,00 & 89,00 & 44,00 & 45,00 & 17,00 \\
\hline $10 \mathrm{BIO} / 40 \mathrm{FC} / 50 \mathrm{CAC}$ & 159,00 & 91,00 & 48,00 & 43,00 & 16,00 \\
\hline $10 \mathrm{BIO} / 30 \mathrm{FC} / 60 \mathrm{CAC}$ & 170,00 & 90,00 & 51,00 & 39,00 & 15,00 \\
\hline $10 \mathrm{BIO} / 20 \mathrm{FC} / 70 \mathrm{CAC}$ & 179,00 & 89,00 & 52,00 & 37,00 & 15,00 \\
\hline
\end{tabular}

SC: substrato comercial à base de casca de pínus e vermiculita; BIO: biossólido; VM: vermiculita média; FC: fibra de coco; CAC: casca de arroz carbonizada.

Para a macroporosidade, apenas os substratos contendo 100\% de CAC, acima de 50\% de CAC combinada com FC, mais de 70\% de CAC combinada com BIO, 20/20/60, 10/40/50, 10/30/60 e 10/20/70 (BIO/FC/CAC) tiveram valores considerados altos (acima de 50\%) por Gonçalves e Poggiani (1996). No entanto, estão próximos da faixa considerada adequada (35-45\%) (Tabela 4).

Quanto à microporosidade, apenas o substrato contendo $100 \%$ de CAC apresentou baixo valor, enquanto que aqueles contendo 10/90, 25/75 (FC/CAC 0,5-1 mm) e 10/90, 25/75 (FC/CAC 0,25-0,5 mm) e aqueles formulados com doses acima de $40 \%$ de BIO combinado com FC/CAC apresentaram alta microporosidade (acima de 55\%) (Tabela 4).

FLORESTA, Curitiba, PR, v. 45, n. 2, p. 393 - 408, abr. / jun. 2015.

Kratz, D. et al.

ISSN eletrônico 1982-4688 / ISSN impresso 0015-3826

DOI: $10.5380 /$ rf.v45i2.31249 
Tabela 5. Correlações entre as propriedades físicas e químicas dos substratos com as variáveis biométricas de mudas de M. scabrella: altura (H150), diâmetro do colo (D150), biomassa seca aérea (BSA), biomassa seca radicial (BSR), agregação das raízes ao substrato (AG) e facilidade de retirada do tubete (FRT) aos 150 dias com densidade aparente $(\mathrm{Da})$, porosidade total $(\mathrm{Pt})$, macroporosidade (Macro), microporosidade (Micro), água facilmente disponível (AFD), matéria orgânica, pH (em água), condutividade elétrica (CE), teor total de sais solúveis (TTSS), potássio (K), cálcio (Ca), magnésio (Mg), fósforo (P), nitrogênio disponível ( $\mathrm{N}$ disp) e enxofre (S).

Table 5. Correlations between the physical and chemical properties of the substrates with the biometric variables of seedlings of $M$. scabrella: height (H150), collar diameter (D150), shoot dry biomass (BSA), root dry biomass BSR), the substrate root aggregation (AG) and ease of removal of the of the tube (FRT) to 150 days with apparent density (Da), total porosity (Pt), macroporosity (Macro) and micro (Micro), readily available water (AFD), organic matter, $\mathrm{pH}$ (in water), electrical conductivity (CE), total content of soluble salts (TTSS), potassium (K), calcium $(\mathrm{Ca})$, magnesium $(\mathrm{Mg})$, phosphorus $(\mathrm{P})$, available nitrogen (N disp) and sulfur $(\mathrm{S})$.

\begin{tabular}{|c|c|c|c|c|c|c|}
\hline & H150 & D150 & BSA & BSR & $\mathbf{A G}$ & FRT \\
\hline $\mathrm{Da}$ & $-0,65^{* * *}$ & $-0,67^{* *}$ & $-0,64^{* *}$ & $-0,64^{* * *}$ & $-0,75^{* *}$ & $0,65^{* *}$ \\
\hline $\mathrm{Pt}$ & $0,10^{\mathrm{ns}}$ & $0,20^{\mathrm{ns}}$ & $0,14^{\mathrm{ns}}$ & $0,17^{\mathrm{ns}}$ & $0,25^{\mathrm{ns}}$ & $-0,13^{\mathrm{ns}}$ \\
\hline Macro & $0,05^{\mathrm{ns}}$ & $0,10^{\mathrm{ns}}$ & $0,10^{\mathrm{ns}}$ & $0,11^{\mathrm{ns}}$ & $0,20^{\mathrm{ns}}$ & $-0,10^{\mathrm{ns}}$ \\
\hline Micro & $-0,02^{n s}$ & $-0,05^{\mathrm{ns}}$ & $-0,06^{\mathrm{ns}}$ & $-0,06^{\mathrm{ns}}$ & $-0,14^{\mathrm{ns}}$ & $0,07^{\mathrm{ns}}$ \\
\hline AFD & $0,32^{\mathrm{ns}}$ & $0,24^{\mathrm{ns}}$ & $0,20^{\mathrm{ns}}$ & $0,19^{\mathrm{ns}}$ & $0,15^{\mathrm{ns}}$ & $-0,26^{\mathrm{ns}}$ \\
\hline MO & $-0,80^{* * *}$ & $-0,76^{* *}$ & $-0,75^{* *}$ & $-0,75^{\text {*** }}$ & $-0,80^{* *}$ & $0,82^{* *}$ \\
\hline $\mathrm{pH}$ & $-0,80^{\text {** }}$ & $-0,80^{\text {*** }}$ & $-0,80^{\text {*** }}$ & $-0,74^{* * *}$ & $-0,81^{* *}$ & $0,79^{\text {** }}$ \\
\hline $\mathrm{CE}$ & $-0,71^{* *}$ & $-0,69^{\text {** }}$ & $-0,66^{* * *}$ & $-0,60^{* *}$ & $-0,71^{* *}$ & $0,68^{* *}$ \\
\hline TTSS & $-0,76^{\text {*** }}$ & $-0,73^{\text {** }}$ & $-0,68^{\text {*** }}$ & $-0,65^{\text {*** }}$ & $-0,77^{* *}$ & $0,72^{\text {** }}$ \\
\hline $\mathrm{K}$ & $0,61^{\text {** }}$ & $0,55^{*}$ & $0,52^{*}$ & $0,53^{*}$ & $0,54^{*}$ & $-0,56^{*}$ \\
\hline $\mathrm{Ca}$ & $0,02^{\mathrm{ns}}$ & $0,07^{\mathrm{ns}}$ & $0,06^{\mathrm{ns}}$ & $-0,04^{\mathrm{ns}}$ & $0,03^{\mathrm{ns}}$ & $0,01^{\mathrm{ns}}$ \\
\hline $\mathrm{Mg}$ & $-0,83^{\text {** }}$ & $-0,79^{* *}$ & $-0,76^{\text {** }}$ & $-0,73^{* *}$ & $-0,82^{* *}$ & $0,81^{* *}$ \\
\hline $\mathrm{P}$ & $0,72^{\text {*** }}$ & $0,72^{* * *}$ & $0,68^{* *}$ & $0,56^{*}$ & $0,72^{* *}$ & $-0,67^{* *}$ \\
\hline $\mathrm{N}$ disp & $0,00^{\mathrm{ns}}$ & $0,03^{\mathrm{ns}}$ & $0,06^{\mathrm{ns}}$ & $0,11^{\mathrm{ns}}$ & $0,09^{\mathrm{ns}}$ & $-0,05^{\mathrm{ns}}$ \\
\hline $\mathrm{S}$ & $-0,58^{*}$ & $-0,55^{*}$ & $-0,51^{*}$ & $-0,53^{*}$ & $-0,61^{* *}$ & $0,57^{*}$ \\
\hline
\end{tabular}

* e **: significativo ao nível de $5 \%$ e $1 \%$ de probabilidade de erro, respectivamente; ${ }^{\text {ns }}$ : não significativo ao nível de $5 \%$ de probabilidade de erro pelo teste $\mathrm{F}$.

Quanto à água facilmente disponível, observou-se uma amplitude de variação de $6 \%$ (CAC) a $45 \%$ (25/75 FC/CAC 0,25-0,5 mm), sendo que os substratos formulados a partir de CAC peneirada apresentaram valores muito superiores aos demais substratos estudados (Tabela 4). Zanetti et al. (2003) observaram que o aumento da granulometria de substratos comerciais à base de FC acarretou diminuição no teor de água disponível, proporcionada pela rápida drenagem em materiais com maior granulometria. Nesses casos, deve-se priorizar uma maior frequência de irrigação, para evitar possível estresse hídrico. Para De Boodt e Verdonck (1972), o teor de água facilmente disponível para as plantas deve ser de 20 a $30 \%$. A partir dessa recomendação, a maioria dos substratos estudados estão localizados dentro da faixa considerada ideal ou então próximos a ela (Tabela 4).

No que se refere ao teor de matéria orgânica, pode-se observar que os substratos à base de BIO apresentaram os maiores valores (de 43,18 a 65,88\%), seguidos do SC, com 48,55\%. Os substratos à base de CAC e FC apresentaram os menores teores de matéria orgânica, variando de 15,25 a 26,92\% (Tabela 6). A matéria orgânica apresentou correlação negativa com o crescimento das mudas de M. scabrella (Tabela 5), porém deve-se observar que o SC apresentou teores de matéria orgânica similares aos dos substratos à base de BIO, logo, não se pode afirmar que apenas os altos teores de matéria orgânica foram prejudiciais ao crescimento das mudas. A resposta apresentada pelas mudas ao teor de matéria orgânica associada ao alto $\mathrm{pH}$ pode estar relacionada com a ecologia da espécie, visto que os bracatingais, segundo Carvalho (1994), ocorrem em solos pobres, ácidos (pH variando entre 3,5 e 5,5) e bem drenados e, conforme pode ser observado na tabela 5, o $\mathrm{pH}$ do substrato comercial foi de 5,47, propiciando um bom crescimento das mudas mesmo com altos teores de matéria orgânica, enquanto que o pH dos substratos à base de BIO variou de 7,76 a 9,06, associado a altos níveis de matéria orgânica.

Quanto ao pH, observa-se que, segundo a recomendação de Gonçalves e Poggiani (1996), apenas o SC, 80/20 e 70/30 (FC/CAC) estão dentro da faixa adequada (5,5-6,5) (Tabela 5), e, segundo a 
recomendação de Kämpf (2005) (5,2 e 5,5), apenas o SC apresentou pH adequado. No entanto, segundo Bailey et al. (2000), quando se trabalha com substratos orgânicos, sem solo, a recomendação é de 4,4 a 6,2 para a maioria dos substratos. Segundo Valeri e Corradini (2000), em substratos com pH abaixo de 5,0 pode ocorrer deficiência de nitrogênio, potássio, cálcio, magnésio e boro, enquanto que em $\mathrm{pH}$ acima de 6,5 são esperadas deficiências de fósforo, ferro, manganês, zinco e cobre.

Tabela 6. Resultados da análise química dos substratos: matéria orgânica (MO), pH (em água), condutividade elétrica $(\mathrm{CE})$, teor total de sais solúveis (TTSS), potássio $(\mathrm{K})$, cálcio $(\mathrm{Ca})$, magnésio $(\mathrm{Mg})$, fósforo $(\mathrm{P})$, nitrogênio disponível ( $\mathrm{N}$ disp) e enxofre ( $\mathrm{S}$ ).

Table 6. Results of chemical analysis of substrates: organic matter (MO), $\mathrm{pH}$ (in water), electrical conductivity (CE), total content of soluble salts (TTSS), potassium (K), calcium (Ca), magnesium $(\mathrm{Mg})$, phosphorus $(\mathrm{P})$, available nitrogen (N disp) and sulfur $(\mathrm{S})$.

\begin{tabular}{|c|c|c|c|c|c|c|c|c|c|c|}
\hline \multirow{2}{*}{ Substratos } & \multirow{2}{*}{$\begin{array}{c}\text { MO } \\
\%\end{array}$} & \multirow{2}{*}{$\begin{array}{c}\mathrm{pH} \\
\mathrm{H}_{2} \mathrm{O}\end{array}$} & \multirow{2}{*}{$\begin{array}{l}\mathrm{CE} \\
\mathrm{mS}\end{array}$} & \multirow{2}{*}{$\begin{array}{l}\text { TTSS } \\
\text { g.L } L^{-1}\end{array}$} & \multirow{2}{*}{\multicolumn{3}{|c|}{$\begin{array}{cc}\mathrm{Ca} & \mathrm{Mg} \\
\mathrm{cmol} / \mathrm{dm}^{3} & \end{array}$}} & $\mathbf{P}$ & N disp & $\mathbf{S}$ \\
\hline & & & & & & & & \multicolumn{3}{|c|}{$\mathrm{mg} / \mathrm{dm}^{3}$} \\
\hline SC & 48,55 & 5,47 & 1,53 & 5,86 & 1,36 & 14,46 & 6,45 & 577,75 & 23,39 & 1705,59 \\
\hline CAC & 24,73 & 8,07 & 0,08 & 0,08 & 1,44 & 0,33 & 0,15 & 66,35 & 19,47 & 3,58 \\
\hline CAC $(0,5-1)$ & 26,92 & 8,11 & 0,23 & 0,38 & 4,29 & 0,89 & 0,53 & 209,00 & 19,22 & 16,03 \\
\hline $\mathrm{CAC}(0,25-0,5)$ & 27,47 & 8,05 & 0,3 & 0,59 & 3,89 & 1,11 & 0,60 & 215,40 & 30,74 & 21,36 \\
\hline $50 \mathrm{CAC}+50 \mathrm{VM}$ & 7,05 & 7,92 & 0,06 & 0,09 & 1,07 & 1,89 & 3,21 & 66,85 & 19,71 & 3,58 \\
\hline $10 \mathrm{FC}+90 \mathrm{CAC}(0,5-1)$ & 26,25 & 7,75 & 0,38 & 0,54 & 3,64 & 1,01 & 0,55 & 146,00 & 20,45 & 25,39 \\
\hline $25 \mathrm{FC}+75 \mathrm{CAC}(0,5-1)$ & 25,42 & 7,47 & 0,49 & 0,65 & 3,55 & 1,69 & 0,70 & 174,00 & 23,39 & 43,32 \\
\hline $10 \mathrm{FC}+90 \mathrm{CAC}(0,25-0,5)$ & 27,26 & 7,77 & 0,41 & 0,70 & 4,00 & 1,44 & 0,76 & 147,25 & 20,69 & 24,59 \\
\hline $25 \mathrm{FC}+75 \mathrm{CAC}(0,25-0,5)$ & 26,22 & 7,33 & 0,62 & 1,00 & 4,36 & 2,14 & 0,71 & 212,75 & 31,97 & 28,23 \\
\hline $80 \mathrm{FC}+20 \mathrm{CAC}$ & 15,25 & 6,23 & 1,09 & 1,12 & 3,55 & 2,94 & 0,79 & 312,75 & 106,01 & 62,42 \\
\hline $70 \mathrm{FC}+30 \mathrm{CAC}$ & 15,68 & 6,39 & 0,96 & 1,21 & 3,11 & 3,44 & 0,86 & 267,75 & 97,92 & 55,64 \\
\hline $60 \mathrm{FC}+40 \mathrm{CAC}$ & 18,39 & 6,70 & 0,73 & 0,90 & 2,18 & 2,85 & 0,60 & 206,05 & 70,71 & 45,17 \\
\hline $50 \mathrm{FC}+50 \mathrm{CAC}$ & 19,83 & 7,12 & 0,54 & 0,63 & 2,69 & 2,61 & 0,56 & 193,50 & 62,37 & 52,56 \\
\hline $40 \mathrm{FC}+60 \mathrm{CAC}$ & 20,59 & 7,00 & 0,62 & 0,76 & 2,23 & 1,69 & 0,45 & 209,90 & 29,27 & 48,56 \\
\hline $30 \mathrm{FC}+70 \mathrm{CAC}$ & 21,63 & 7,06 & 0,62 & 0,74 & 1,95 & 1,16 & 0,34 & 190,45 & 24,12 & 69,20 \\
\hline $20 \mathrm{FC}+80 \mathrm{CAC}$ & 21,89 & 7,59 & 0,36 & 0,41 & 1,30 & 1,11 & 0,44 & 83,50 & 21,18 & 73,51 \\
\hline $50 \mathrm{BIO}+50 \mathrm{CAC}$ & 62,81 & 8,75 & 2,74 & 8,78 & 0,55 & 2,80 & 14,34 & 18,65 & 31,48 & 926,21 \\
\hline $40 \mathrm{BIO}+60 \mathrm{CAC}$ & 61,80 & 8,88 & 2,34 & 6,90 & 0,65 & 3,01 & 15,94 & 13,75 & 30,25 & 904,65 \\
\hline $30 \mathrm{BIO}+70 \mathrm{CAC}$ & 54,08 & 8,79 & 1,67 & 4,34 & 0,74 & 2,90 & 11,55 & 10,70 & 27,80 & 841,50 \\
\hline $20 \mathrm{BIO}+80 \mathrm{CAC}$ & 51,70 & 9,06 & 1,25 & 2,67 & 0,97 & 2,31 & 8,14 & 27,55 & 26,82 & 285,76 \\
\hline $10 \mathrm{BIO}+90 \mathrm{CAC}$ & 43,18 & 9,13 & 0,85 & 1,48 & 0,90 & 1,44 & 3,08 & 107,80 & 26,82 & 96,00 \\
\hline $50 \mathrm{BIO}+50 \mathrm{FC}$ & 62,38 & 8,81 & 2,66 & 8,58 & 1,75 & 3,49 & 18,41 & 26,30 & 59,67 & 1274,32 \\
\hline $50 \mathrm{BIO}+40 \mathrm{FC}+10 \mathrm{CAC}$ & 65,88 & 8,96 & 2,72 & 9,24 & 1,58 & 3,33 & 17,30 & 25,20 & 53,30 & 1349,79 \\
\hline $50 \mathrm{BIO}+30 \mathrm{FC}+20 \mathrm{CAC}$ & 64,73 & 8,91 & 2,66 & 8,90 & 1,32 & 3,48 & 17,70 & 16,40 & 52,32 & 1143,39 \\
\hline $50 \mathrm{BIO}+20 \mathrm{FC}+30 \mathrm{CAC}$ & 64,91 & 8,89 & 2,61 & 9,09 & 1,03 & 3,18 & 16,16 & 15,00 & 57,22 & 1015,55 \\
\hline $40 \mathrm{BIO}+50 \mathrm{FC}+10 \mathrm{CAC}$ & 62,02 & 8,67 & 2,34 & 6,98 & 1,55 & 3,21 & 17,54 & 21,55 & 40,06 & 910,81 \\
\hline $40 \mathrm{BIO}+40 \mathrm{FC}+20 \mathrm{CAC}$ & 60,44 & 8,34 & 2,12 & 6,48 & 1,25 & 3,33 & 16,50 & 39,75 & 57,96 & 963,18 \\
\hline $40 \mathrm{BIO}+30 \mathrm{FC}+30 \mathrm{CAC}$ & 58,82 & 8,31 & 2,35 & 7,13 & 1,20 & 2,84 & 13,99 & 28,65 & 49,13 & 909,27 \\
\hline $40 \mathrm{BIO}+20 \mathrm{FC}+40 \mathrm{CAC}$ & 60,28 & 8,35 & 2,26 & 6,85 & 1,21 & 2,91 & 14,49 & 14,40 & 46,68 & 804,53 \\
\hline $30 \mathrm{BIO}+50 \mathrm{FC}+20 \mathrm{CAC}$ & 55,99 & 8,21 & 1,99 & 4,91 & 2,00 & 3,19 & 11,96 & 34,95 & 43,74 & 733,68 \\
\hline $30 \mathrm{BIO}+40 \mathrm{FC}+30 \mathrm{CAC}$ & 56,61 & 8,19 & 1,92 & 5,11 & 1,95 & 3,09 & 11,58 & 35,55 & 39,81 & 764,48 \\
\hline $30 \mathrm{BIO}+30 \mathrm{FC}+40 \mathrm{CAC}$ & 58,63 & 8,89 & 2,03 & 5,30 & 1,43 & 2,88 & 11,45 & 35,75 & 47,66 & 727,52 \\
\hline $30 \mathrm{BIO}+20 \mathrm{FC}+50 \mathrm{CAC}$ & 57,94 & 9,01 & 1,98 & 5,26 & 1,17 & 2,58 & 10,53 & 22,35 & 40,80 & 519,58 \\
\hline $20 \mathrm{BIO}+50 \mathrm{FC}+30 \mathrm{CAC}$ & 49,57 & 8,81 & 1,93 & 4,58 & 2,01 & 3,34 & 9,89 & 147,75 & 41,78 & 454,89 \\
\hline $20 \mathrm{BIO}+40 \mathrm{FC}+40 \mathrm{CAC}$ & 51,04 & 8,9 & 1,85 & 4,38 & 1,70 & 2,86 & 11,33 & 125,20 & 41,29 & 360,93 \\
\hline $20 \mathrm{BIO}+30 \mathrm{FC}+50 \mathrm{CAC}$ & 51,29 & 9,01 & 1,65 & 3,66 & 1,73 & 2,68 & 8,46 & 84,70 & 44,23 & 575,03 \\
\hline $20 \mathrm{BIO}+20 \mathrm{FC}+60 \mathrm{CAC}$ & 48,62 & 9,04 & 1,7 & 4,02 & 1,16 & 2,48 & 7,59 & 135,20 & 40,80 & 396,35 \\
\hline $10 \mathrm{BIO}+50 \mathrm{FC}+40 \mathrm{CAC}$ & 50,12 & 7,76 & 1,34 & 2,45 & 2,18 & 2,78 & 6,25 & 157,00 & 44,72 & 216,14 \\
\hline $10 \mathrm{BIO}+40 \mathrm{FC}+50 \mathrm{CAC}$ & 44,79 & 8,12 & 1,24 & 2,21 & 2,13 & 2,69 & 7,36 & 211,40 & 34,67 & 214,60 \\
\hline $10 \mathrm{BIO}+30 \mathrm{FC}+60 \mathrm{CAC}$ & 48,90 & 8,32 & 1,23 & 2,28 & 1,68 & 2,60 & 6,93 & 153,70 & 33,69 & 272,82 \\
\hline $10 \mathrm{BIO}+20 \mathrm{FC}+70 \mathrm{CAC}$ & 45,76 & 8,51 & 1,12 & 2,10 & 1,41 & 1,98 & 7,38 & 166,65 & 61,14 & 189,34 \\
\hline
\end{tabular}

SC: substrato comercial à base de casca de pínus e vermiculita; BIO: biossólido; VM: vermiculita média; FC: fibra de coco; CAC: casca de arroz carbonizada.

$\mathrm{O}$ aumento do $\mathrm{pH}$ teve efeito negativo para a altura $(\mathrm{R}=-0,80)$, diâmetro de colo $(\mathrm{R}=-0,80)$, biomassa seca aérea $(R=-0,80)$, biomassa seca radicial $(R=-0,74)$, facilidade de retirada do tubete

FLORESTA, Curitiba, PR, v. 45, n. 2, p. 393 - 408, abr. / jun. 2015.

Kratz, D. et al.

ISSN eletrônico 1982-4688 / ISSN impresso 0015-3826

403

DOI: $10.5380 /$ rf.v45i2.31249 
$(\mathrm{R}=0,79)$ e agregação das raízes ao substrato $(\mathrm{R}=-0,81)$ (Tabela 4). Deve-se enfatizar que o $\mathrm{pH}$ influencia indiretamente nas variáveis facilidade de retirada do tubete e agregação das raízes ao substrato, visto que elas estão relacionadas à qualidade do sistema radicial e às propriedades físicas dos substratos. Neste estudo, as mudas de M. scabrella que apresentaram maior crescimento foram produzidas em $\mathrm{pH}$ variando de 5,47 (SC) a 7,59 (20 FC / 80 CAC) (Tabela 1 e 5).

Verifica-se, na tabela 5, que o aumento da dose de BIO acarreta aumento da condutividade elétrica e do teor total de sais solúveis, lembrando que a salinidade é influenciada pela condutividade elétrica. Resultado semelhante foi observado por Guerrini e Trigueiro (2004), resposta que, segundo os autores, está diretamente ligada à alta concentração de micronutrientes encontrados no biossólido.

Quanto à salinidade, segundo a classificação de Röber e Schaller (1985), observa-se na tabela 5 que o SC apresentou salinidade extremamente alta $\left(5,86 \mathrm{~g} . \mathrm{L}^{-1}\right)$ e que parte dos substratos contendo BIO apresentaram salinidade considerada tóxica $\left(>7,0\right.$ g. $\left.\mathrm{L}^{-1}\right)$, extremamente alta $\left(5,0\right.$ a 7,0 g. $\left.\mathrm{L}^{-1}\right)$, muito alta $\left(4,0\right.$ a 5,0 g. $\left.\mathrm{L}^{-1}\right)$ e alta $\left(2,0\right.$ a 4,0 g.. $\left.\mathrm{L}^{-1}\right)$. Já os substratos que continham apenas FC e CAC apresentaram salinidade dentro da faixa considerada baixa $\left(<1,0\right.$ g. $\left.\mathrm{L}^{-1}\right)$ e normal $\left(1,0\right.$ a 2,0 g. $\left.\mathrm{L}^{-1}\right)$. No que se refere ao $\mathrm{SC}$, cabe ressaltar que ele recebeu adubação química no momento de sua fabricação.

A salinidade apresentou correlações negativas com a altura $(R=-0,76)$, com o diâmetro de colo $(R=-0,73)$, com a biomassa seca aérea $(R=-0,68)$ e com a biomassa seca radicial $(R=-0,65)$ (Tabela 4$)$. $\mathrm{O}$ menor crescimento das mudas nos substratos com alta condutividade elétrica e salinidade está relacionado ao fato de que altos valores de condutividade elétrica, representados por níveis altos de salinidade, podem danificar as raízes e os pelos radiciais, impedindo a absorção de água e nutrientes, afetando a atividade fisiológica e consequentemente diminuindo o crescimento das plantas (RODRIGUES, 2002).

Os resultados das análises de nutrientes apresentados na tabela 6 mostram que a presença do componente $\mathrm{BIO}$ acarreta aumento na concentração de $\mathrm{Ca}, \mathrm{Mg}$ e $\mathrm{S}$ e diminuição na concentração de $\mathrm{P}$ e K. Enquanto a FC apresentou maior concentração de $\mathrm{N}$ disponível e P, a CAC apresentou baixas concentrações de todos os elementos analisados. Resultados semelhantes foram encontrados por Guerrini e Trigueiro (2004), com o acréscimo de BIO à CAC ocasionando aumento da concentração de N, P, Ca, Mg e S. Neste estudo, o biossólido apresentou baixa concentração de P, comprovando assim que é um material heterogêneo e que sua composição varia segundo as características dos esgotos e sua forma de tratamento.

Segundo a recomendação de Gonçalves e Poggiani (1996), a maioria dos substratos analisados apresentou baixa concentração de $\mathrm{K}\left(<1,5 \quad \mathrm{cmol}_{\mathrm{c}} \cdot \mathrm{dm}^{-3}\right)$, sendo que apenas aqueles contendo concentrações de até $70 \%$ de CAC combinada com FC apresentaram níveis adequados (3,0-10,0 $\left.\mathrm{cmol}_{\mathrm{c} .} \mathrm{dm}^{-3}\right)$. Verificou-se correlação positiva do $\mathrm{K}$ com altura $(\mathrm{R}=0,61)$, diâmetro de colo $(\mathrm{R}=0,55)$, biomassa seca aérea $(R=0,52)$, biomassa seca radicial $(R=0,52)$ e agregação das raízes ao substrato $(\mathrm{R}=0,53)$, e correlação negativa com a facilidade de retirada do tubete $(\mathrm{R}=-0,56)$, indicando que esse elemento é fundamental para o crescimento da parte aérea e radicial (Tabela 5).

A concentração de Ca foi adequada apenas para o SC, provavelmente porque este recebeu adubação química no momento de sua fabricação. Os demais substratos analisados apresentaram baixa concentração $\left(<10 \mathrm{cmol}_{\mathrm{c}} \cdot \mathrm{dm}^{-3}\right)$, segundo Gonçalves e Poggiani (1996). Os substratos formulados a partir de $20 \%$ de BIO apresentaram altos níveis de $\mathrm{Mg}\left(>10 \mathrm{cmol}_{\mathrm{c}} \cdot \mathrm{dm}^{-3}\right)$ e doses inferiores a $20 \%$ de BIO combinado com FC/CAC e o SC enquadraram-se na faixa considerada adequada $\left(5-10 \mathrm{cmol}_{\mathrm{c}} \cdot \mathrm{dm}^{-3}\right)$, enquanto que os tratamentos formulados com apenas FC/CAC mostraram baixos níveis de $\mathrm{Mg}$ $\left(<5 \mathrm{cmol}_{\mathrm{c} .} \mathrm{dm}^{-3}\right)$, segundo a recomendação de Gonçalves e Poggiani (1996). O Mg apresentou correlação negativa com altura $(R=-0,83)$, diâmetro de colo $(R=-0,79)$, biomassa seca aérea $(R=-0,76)$ e biomassa seca radicial $(R=-0,73)$ (Tabela 5). Esse fato pode estar ligado à alta concentração de $\mathrm{Mg}$ nos substratos à base de BIO, que foi um dos fatores que prejudicaram o crescimento das mudas.

A concentração de $\mathrm{P}$ foi adequada apenas para o SC, tendo os demais substratos analisados apresentado baixa $\left(<200 \mathrm{mg} \cdot \mathrm{dm}^{-3}\right)$ e média $\left(200-400 \mathrm{mg} \cdot \mathrm{dm}^{-3}\right)$ concentração, segundo Gonçalves e Poggiani (1996). O P apresentou correlação positiva com crescimento em altura $(\mathrm{R}=0,72)$, diâmetro de colo $(R=0,72)$ e biomassa seca aérea $(R=0,68)$ das mudas, indicando que esse elemento é fundamental para o crescimento da parte aérea (Tabela 5). No substrato, esse elemento é imóvel (MARSCHNER, 1995), fazendo com que, em concentrações mais baixas, as raízes tenham que se deslocar para absorvê-lo, resultando em maior crescimento das mesmas. 
Quanto ao $\mathrm{S}$, o $\mathrm{SC}$ e os formulados à base de $\mathrm{BIO}$ apresentaram as maiores concentrações desse elemento, quando comparados com os substratos à base de FC e CAC (Tabela 6). Verificou-se correlação negativa com altura $(R=-0,58)$, diâmetro de colo $(R=-0,55)$, biomassa seca aérea $(R=-0,51)$ e biomassa seca radicial $(R=-0,53)$, indicando, assim, que, quanto maior a concentração de $S$, menor o crescimento das mudas (Tabela 5). Deve-se observar, porém, que as mudas produzidas no SC apresentaram um bom crescimento, e esse substrato apresentou concentrações de S superiores às do BIO, de modo que não se pode afirmar que esse elemento foi prejudicial ao crescimento das mudas de M. scabrella.

Deve-se ressaltar que a fertilidade do substrato no momento do cultivo foi diferente da presente análise, visto que ela foi realizada nos substratos sem adubação, tendo como objetivo verificar se a concentração dos macronutrientes não estava excessiva ou tóxica. Conforme observado, a maioria dos elementos apresentou baixa concentração, o que pode ser corrigido com adubação de base e cobertura. Dessa forma, os teores de macronutrientes presentes não podem ser utilizados para justificar o crescimento das mudas, salvo se os substratos receberam fertilização após o seu preparo.

\section{CONCLUSÕES}

- $\quad \mathrm{O}$ SC e as diferentes combinações de CAC e FC apresentaram-se viáveis para a produção de mudas de M. scabrella, enquanto que o componente BIO apresentou-se viável apenas em pequenas concentrações.

- O peneiramento da CAC não se justifica, quando comparado com a CAC na sua forma íntegra.

- Densidade, matéria orgânica, $\mathrm{pH}$, condutividade elétrica, salinidade e concentração de magnésio, potássio e fósforo influenciaram no crescimento das mudas.

\section{REFERÊNCIAS}

BAILEY, D. A.; FONTENO, W. C.; NELSON, P. V. Greenhouse substrates and fertilization. Raleigh: North Caroline State University, 2000. Disponível em: <http://www.ces.ncsu.edu/depts/hort/floriculture/ plugs/ghsubfert.pdf.>. Acesso em 11/04/2011.

BIANCHETTI, A. Comparação de tratamentos para superar a impermeabilidade do tegumento de sementes de bracatinga (Mimosa scabrella Benth.). Boletim de Pesquisa Florestal, Curitiba, v. 2, p. 57$68,1981$.

BONNET, B. R. P. Produção de mudas de Eucalyptus viminalis Lambill. (Myrtaceae), Schinus terebinthifolius Raddi (Anacardiaceae) e Mimosa scabrella Benth. (Mimosaceae) em substrato com lodo de esgoto anaeróbio digerido alcalinizado e compostado. $135 \mathrm{f}$. Dissertação (Mestrado em Ciências Florestais) - Universidade Federal do Paraná, Curitiba, 2001.

CARNEIRO, J. G. de A. Produção e controle de qualidade de mudas florestais. Curitiba: UFPR/FUPEF, 1995. $451 \mathrm{p}$.

CARVALHO, P. E. R. Mimosa scabrella Benth.: bracatinga. In: CARVALHO, P. E. R. Espécies florestais brasileiras: recomendações silviculturais, potencialidades e uso da madeira. Colombo: EMBRAPA-CNPF/Brasília: EMBRAPA-SPI, p. 337-343, 1994.

CUNHA, A. M.; CUNHA, G. M.; SARMENTO, R. A.; CUNHA, G. M.; AMARAL, J. F. T. Efeito de diferentes substratos sobre o desenvolvimento de mudas de Acacia sp. Revista Árvore, v. 30, n. 2, p. 207-214, 2006.

DE BOODT, M.; VERDONCK, O. The physical properties of the substrates in: Floriculture. Acta Horticulturae, Wageningen, v. 26, p. 37-44, 1972.

EMPRESA BRASILEIRA DE PESQUISA AGROPECUÁRIA (EMBRAPA). Manual técnico da bracatinga (Mimosa scabrella Benth.). Colombo: EMBRAPA-CNPF, 1988. 70 p. (EMBRAPA-CNPF. Documentos, 20).

FERMINO, M. H. Métodos de análise para caracterização física de substratos. 89 p. Tese (Doutorado em Fitotecnia) - Universidade Federal do Rio Grande do Sul, Porto Alegre, 2003. 
GOMES, J. M.; PAIVA, H. N. Viveiros florestais (propagação sexuada). Viçosa: Editora UFV, 2004. (Caderno didático, 72).

GONÇALVES, J. L. M.; SANTARELLI, E. G.; MORAES NETO, S. P.; MANARA, M. P. Produção de mudas de espécies nativas: substrato, nutrição, sombreamento e fertilização. In: GONÇALVES, J. L. M.; BENEDETTI, V. (Eds.). Nutrição e fertilização florestal. Piracicaba: Ipef, 2000. p. 309-350.

GONÇALVES, L. M.; POGGIANI, F. Substratos para produção de mudas florestais. In: CONGRESSO LATINO AMERICANO DE CIÊNCIA DO SOLO, 13. Águas de Lindoia, 1996. Resumos... Piracicaba, Sociedade Latino Americana de Ciência do Solo, CD-ROM, 1996.

GUERRINI, I. A.; TRIGUEIRO, R. M. Atributos físicos e químicos de substratos compostos por biossólidos e casca de arroz carbonizada. Revista Brasileira de Ciência do Solo, Viçosa, v. 28, p. 10691076, 2004.

KÄMPF, A. N. Substrato. In: KAMPF, A. N. Produção comercial de plantas ornamentais. 2. ed. Guaíba: Agrolivros, 2005. p. 45-72.

KNAPIK, J. G.; ALMEIDA, L. S.; FERRARI, M. P.; OLIVEIRA, E. B.; NOGUEIRA, A. C. Crescimento inicial de Mimosa scabrella Benth. (Bracatinga), Schinus terebinthifolius Raddi (Aroeira) e Allophylus Edulis (St.-Hil.) Radl. (Vacum) sob diferentes regimes de adubação. Boletim de Pesquisa Florestal, Colombo, n. 51, p. 33-44, 2005.

KRATZ, D.; WENDLING, I.; NOGUEIRA, A. C.; SOUZA, P. V. D. de. Substratos renováveis na produção de mudas de Eucalyptus benthamii. Ciência Florestal, Santa Maria, v. 23, n. 4, p. 607-621, 2013.

MARSCHNER, H. Mineral nutrition of higher plants. 2nd ed. San Diego: Academic Press, 1995, 889 p.

MINISTÉRIO DA AGRICULTURA, PECUÁRIA E ABASTECIMENTO (MAPA). Instrução Normativa SDA no 17. Diário Oficial da União - Seção 1, n 99, 24 de maio de 2007. Métodos Analíticos Oficiais para Análise de Substratos para Plantas e Condicionadores de Solo. Brasília, 2007.

NÓBREGA, R. S.; BOAS, R. C. V.; NÓBREGA, J. C. A.; PAULA, A. M.; MOREIRA, F. M. S. Utilização de biossólido no crescimento inicial de mudas de aroeira (Schinus terebinthifolius Raddi). Revista Árvore, Viçosa, v. 31, n. 2, 2007.

NOGUEIRA, A. R. de A.; SOUZA, G. B. de (Eds.). Manual de laboratórios: solo, água, nutrição vegetal, nutrição animal e alimentos. São Carlos: Embrapa Pecuária Sudeste, 2005.

NOGUERA, P.; ABAD, M.; NOGUERA, V.; PUCAHDES, R.; MAQUIEIRA, A. Coconut coir waste, a new viable ecologilly - Friendly peat substitute. Acta Horticulturae, Wageningen, v. 517, p. 279-286, 2000.

RÖBER, R.; SCHALLER, K. Plantzenernährung im Gerbau. 3. ed. Sttugart: Ulmer, 1985. 352 p.

RODRIGUES, L. R. F. Técnicas de cultivo hidropônico e de controle ambiental no manejo de pragas, doenças e nutrição vegetal em ambiente protegido. Jaboticabal: FUNEP, 2002, 762 p.

ROTTA, E.; OLIVEIRA, Y. M. M. de. Área de distribuição natural da bracatinga (Mimosa scabrella). In: SEMINÁRIO SOBRE ATUALIDADES E PERSPECTIVAS FLORESTAIS, 4.: Bracatinga, uma alternativa para reflorestamento, Curitiba, 1981. Anais... Curitiba, EMBRAPA-URPFCS, 1981. p. 1-24.

SCHMITZ, J. A. K.; SOUZA, P. V. D.; KÄMPF, A. N. Propriedades químicas e físicas de substratos de origem mineral e orgânica para o cultivo de mudas em recipientes. Ciência Rural, Santa Maria, v. 32, n. 6, p. 937-944, 2002.

STURION, J. A. Produção de mudas de Mimosa scabrella Benth. In: SEMINÁRIO SOBRE ATUALIDADES E PERSPECTIVAS FLORESTAIS, 4.: Bracatinga, uma alternativa para reflorestamento, Curitiba, 1981. Anais... Curitiba, EMBRAPA-URPFCS, 1981. p. 39-52.

TRIGUEIRO, R. de M. Uso de biossólidos como substrato para produção de mudas de pínus e eucalipto. 94 F. Dissertação (Mestrado em Agronomia) - Universidade Estadual Paulista "Júlio De Mesquita Filho", Botucatu, SP, 2002. 
TRIGUEIRO, R. de M.; GUERRINI, I. A. Uso de biossólido como substrato para produção de mudas de eucalipto. Scientia Forestalis, Piracicaba, v. 64, p. 150-162, 2003.

VALERI, S. V.; CORRADINI, L. Fertilização em viveiros para produção de mudas de Eucalyptus e Pinus. In: GONÇALVES, J. L. M.; BENEDETTI, V. (Eds.). Nutrição e fertilização florestal. Piracicaba: IPEF, 2000. p. 167-189.

WEBER, K. S. Manejo da bracatinga (Mimosa scabrella Benth.) baseado no crescimento diamétrico de árvores individuais. 125 f. Dissertação (Mestrado em Ciências Florestais) - Universidade Federal do Paraná, Curitiba, 2007.

WENDLING, I.; DUTRA, L. F. Produção de mudas de eucalipto por sementes. In: WENDLING, I.; DUTRA, L. F. Produção de mudas de eucalipto. Colombo: Embrapa Florestas, 2010. p. 13-47.

WENDLING, I.; GUASTALA, D.; DEDECEK, R. Características físicas e químicas de substratos para produção de mudas de Ilex paraguariensis St.-Hil. Revista Árvore, Viçosa, v. 31, p. 209-220, 2007.

ZANETTI, M.; FERNANDES, C.; CAZETTA, J. O.; CORA, J. E.; MATTOS JÚNIOR, D. Características físicas de substratos para produção de mudas cítricas sob telado. Laranja, Cordeirópolis, v. 24, n. 2, p. 507-518, 2003. 
FLORESTA, Curitiba, PR, v. 45, n. 2, p. 393 - 408, abr. / jun. 2015. Kratz, D. da et al. 